\title{
Petrography of the Neogene Siwalik Group sandstones, Khutia Khola section, Nepal Himalaya: implications for provenance, paleoclimate and tectonic setting
}

\author{
*Swostik Kumar Adhikari and Tetsuya Sakai \\ Department of Geoscience, Shimane University, Matsue 690-8504, Shimane, Japan \\ *(Corresponding email: swostik_adhikari@hotmail.com)
}

\begin{abstract}
The Neogene fluvial sediment (Siwalik Group) forming the southernmost foothill in the Himalaya is one of the best archives of climate history and uplift of the Himalaya. The petrographic composition of these fluvial sandstones provides information on source rocks, depositional climate of the Siwalik sediments and tectonic setting of the Himalaya. This study presents the petrographical results of the Siwalik sandstones from the Khutia Khola section, Nepal Himalaya. Petrographically, these sandstones are lithic arenites (30\%) to sub-litharenites (70\%). Provenance analyses show that sediments were mainly derived from recycled orogenic and transitional recycled areas in the recycled orogen block provenances. The climatic indexes suggest the semi-humid to humid climate during sedimentation of the Khutia Khola sandstones. The stratigraphic trend of the lithic components show that metamorphic lithic grains are increasing and sedimentary lithic grains are decreasing in up section, and the Lesser and Higher Himalayas are the mixed source of these Siwalik sandstones.
\end{abstract}

Keywords: Siwalik Group, Khutia Khola, Petrography, Provenance, Sandstone

Received: 13 January 2017

Revision accepted: 1 May 2017

\section{INTRODUCTION}

Petrography of sandstone deciphers the nature of the source rocks (Pettijohn 1975; Folk 1980; Blatt et al. 1980), tectonic setting of the source area (Dickinson 1970, 1985; Crook 1974; Basu et al. 1975; Dickinson and Suczek 1979; Ingersoll and Suczek 1979; Dickinson et al. 1983) and climatic condition during sedimentation (Suttner et al. 1981; Suttner and Dutta 1986; Weltje 1994, 2002; Weltje et al. 1998). At the same time, weathering conditions in the source area also control the sediments composition (Critelli et al. 1997). In tectonically active area, source rock type determines sediment composition (Dickinson 1970; Dickinson et al. 1983) however, climate and relief play important roles in determining the final composition of sediments in tectonically less active area (Basu 1976; Suttner et al. 1981). Such methods have been used by several researches for study of the Siwalik sandstones to reveal the provenance and tectonic history of the Himalaya (cf. Hisatomi 1990; Tokuoka et al. 1986, 1988; Critelli and Ingersoll 1994; Dhital et al. 1995; DeCelles et al. 1998; Tamrakar et al. 2003; Sigdel and Sakai 2013; Tamrakar and Syangbo 2014)

This study focuses on the sandstone petrography of the Neogene Siwalik Group in the Khutia Khola section of Nepal Himalaya (Fig. 1). The Khutia Khola section is previously studied as one of the sites among several river sections of the Siwalik Group in the Nepal Himalaya and is focused in terms of isotopes, age dating and petrography (Quade et al. 1995; DeCelles et al. 1998; Ojha et al. 2000). The purpose of this paper is to describe the petrography of the Neogene Siwalik sandstones from the Khutia Khola section of Nepal Himalaya in detail, and determine the provenance, paleoclimate and tectonic settings.

\section{GEOLOGICAL SETTING}

The Siwalik Group at the southern front of the entire Himalayan belt yielded in association with Himalayan uplift (Gansser 1964; Prakash et al. 1980; Burbank et al. 1996; DeCelles et al. 1998; Upreti 1999). The Siwalik Group is demarked by the Main Boundary Thrust (MBT) from the Lesser Himalaya to the north and the Main Frontal Thrust (MFT) from the Indo-Gangatic Plain to the south (Nakata 1989; Mugnier et al. 1999). In general, the Main Dun Thrust (MDT) separates the Siwalik Group into the southern and northern belts (DeCelles et al. 1998; Robinson et al. 2006; Tokuoka et al. 1986; Ulak and Nakayama 1998). In the study area, the Jogbudha Thrust (JT) equivalent to MDT and Rangun Khola Thrust (RKT) separate the Siwalik Group into the southern, central and northern belts (Sharma et al. 2007).

The Siwalik Group has been traditionally classified in three units (Lower, Middle and Upper Siwaliks) (Auden 1935; Hagen 1969; Yoshida and Arita 1982; Quade et al. 1995; DeCelles et al. 1998; Gautam and Fujiwara 2000; Ojha et al. 2000; Robinson et al. 2006; Sharma et al. 2007) and locally two-, four- or five- fold units are also acceptable (Glennie and Ziegler 1964; Sharma 1973; Tokuoka et al. 1986, 1988; Sah et al. 1994; Corvinus and Nanda 1994; Dhital et al. 1995; Ulak and Nakayama 1998; Sigdel et al. 2011). The common three-fold classification starts in general with the mudstonedominated Lower Siwalik, grading upward into the sandstonedominated Middle Siwalik and the Upper Siwalik of the conglomerate-dominated sediments (Medlicott 1875; Pilgrim 1913; Wadia 1957; Auden 1935; Hagen 1969; Yoshida and Arita 1982; Quade et al. 1995; DeCelles et al. 1998; Upreti 1999; Gautam and Fujiwara 2000; Ojha et al. 2000; Robinson 


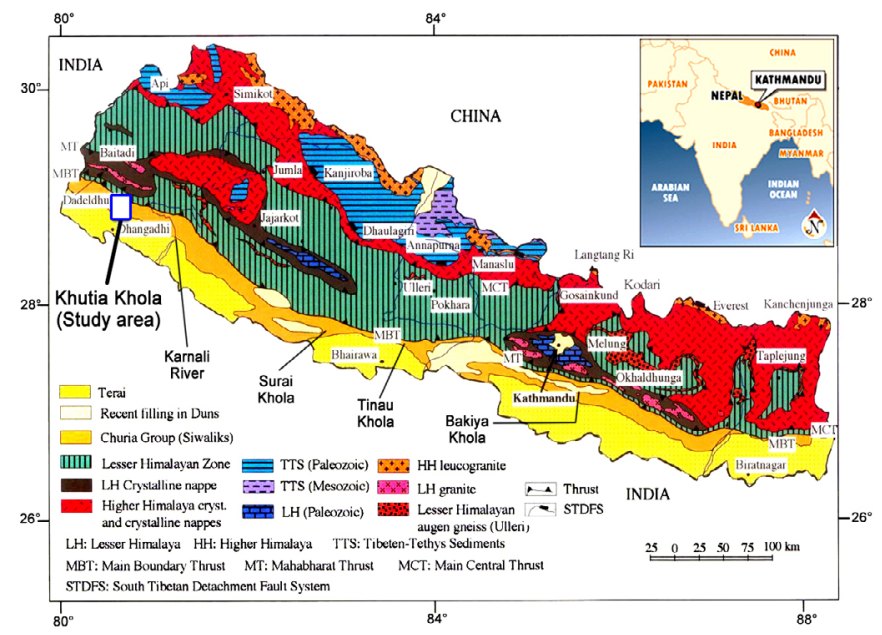

Fig. 1: Location map of the study area. The Khutia Khola area is indicated in box (modified from Upreti and Le Fort 1999).

et al. 2006). The previous studies of the Siwalik Group along the Khutia Khola also followed the classical tripartite classification (DeCelles et al. 1998; Ojha et al. 2000; Sharma et al. 2007). Recently, Adhikari and Sakai (2015) established lithostratigraphy of the southern belt in the area which consists of the Jagati Formation $(2,110 \mathrm{~m}$, equivalent to the Lower Siwalik) and the Kala Formation $(2,050 \mathrm{~m}$, equivalent to the Middle Siwalik) in ascending order (Fig. 2). The Upper Siwalik is not exposed in the area and it may be masked by the Jogbudha Thrust, thrusting the central block onto the southern belt rock from the north. Each formation is further subdivided into three members; the lower, middle and upper members.

The Jagati Formation is composed of variegated, reddishbrown, yellowish-brown, brown, yellowish-grey, grey to greenish-grey mudstones and very fine- to coarse-grained, brown, reddish-grey, light-grey, grey to greenish-grey sandstones. Most of the mudstones show typical characteristic of paleosols containing burrows, rhizoliths, desiccation cracks, nodules, and some mudstones are thinly laminated or massive. The Kala Formation comprises thin- to thickbedded, medium- to very coarse-grained, light-grey, grey to greenish-grey sandstones and pebbly sandstones interbedded with reddish-brown, brown, grey, greenish-grey to dark-grey mudstones. Biotite, quartz and feldspar are abundantly present in sandstones showing "salt and pepper" characteristic. In the lower portion of the upper member sub-rounded to rounded pebbles (1-2 cm diameter) are present in sandstones, whereas gravel size tends to be larger and some cobble sized gravels also appear in the upper portion of this member. Finely laminated or massive mudstones are common, whereas paleosols are infrequent.

\section{FLUVIAL FACIES AND DEPOSITIONAL ENVIRONMENT}

Adhikari (2014) studied fluvial facies and depositional environment in the Khutia Khola section and reconstructed

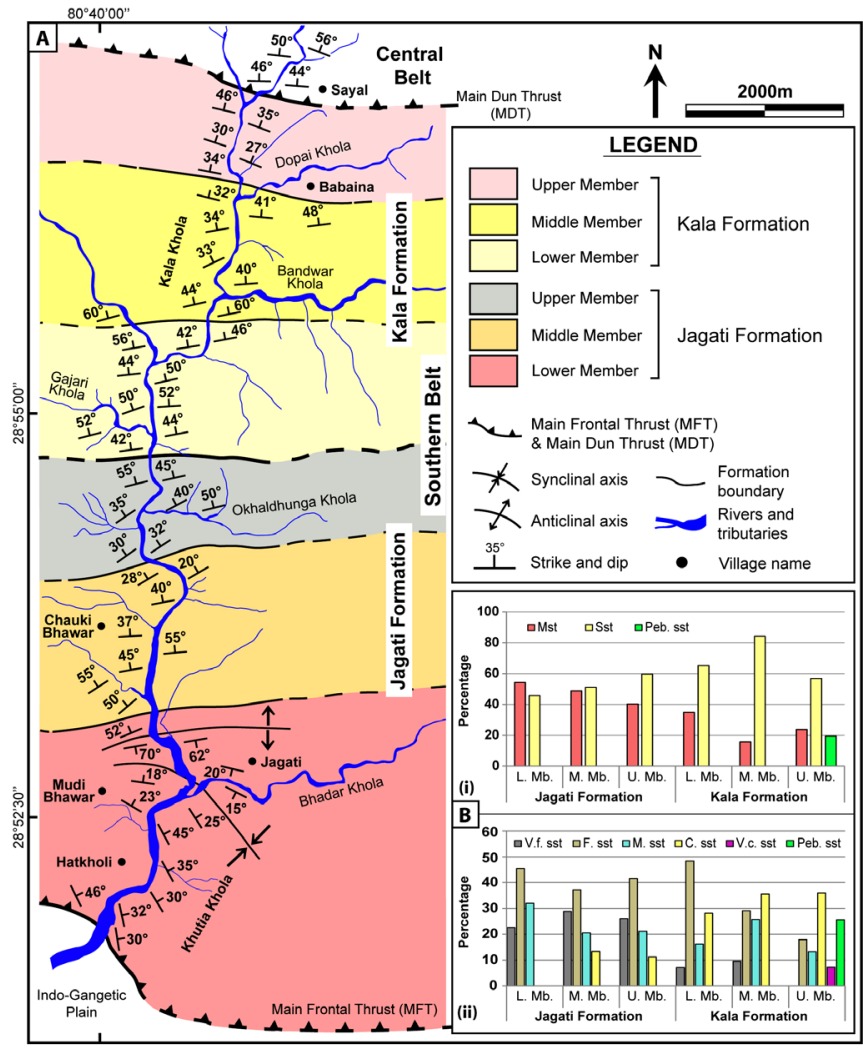

Fig. 2: A. Geological map of the Siwalik Group along the Khutia Khola section. B. (i) Distribution of various rocks. (ii) Distribution of various sandstones. Abbreviation: Mst -mudstone, Sst-sandstone, Peb. sst-pebbly sandstone, V.f. sst-very fine-grained sandstone, F. sst-fine-grained sandstone, $M$. sst-medium grained sandstone, C. sstcoarse-grained sandstone, V.c. sst-very coarse-grained sandstone, L. Mb.-lower member, M. Mb.--middle member, U. Mb.-upper member.

meandering and braided river systems, as have been recognized in the other Siwalik sections in Nepal Himalaya (cf. Nakayama and Ulak 1999; Ulak and Nakayama 2001; Ulak 2004, 2009; Huyghe et al. 2005; Sigdel and Sakai 2016). Each fluvial system are made up of two facies associations; Fine-grained meandering river system (FA1) and Flood-flow dominated meandering river system (FA2), and Deep sandy braided river system (FA3) and, Shallow sandy braided river system (FA4), respectively (Table 1). The facies associations are also more or less correspond to stratigraphic divisions, with FA1 being the lower member and up to the middle part of the middle member of Jagati Formation, and FA2 being the remainder of the middle member and the upper member. Similarly, FA3 corresponds to the lower and middle member of the Kala Formation, and FA4 to the upper member. In the study area, Ojha et al. (2000) analyzed the magnetostratigraphy and the rock units were dated from 13.30 to $7.65 \mathrm{Ma}$. The age of change in the river systems from fine-grained meandering river system (FA1) to flood-flow dominated meandering river system (FA2) is inferred to be before 13.3 Ma, probably around 13.5 Ma. Similarly, the age of change from meandering river system (FA1-FA2) to braided river system (FA3-FA4) is at around 11.0 Ma. 
Table 1: Description of the facies associations recognized in the Khutia Khola section. Abbreviation: FA - facies association, P-paleosol, Fl-laminated mudstone, Fm-massive mudstone, Sp-planar-cross-bedded sandstone, St-trough-crossbedded sandstone, Sr-ripple cross-laminated sandstone, Sh-horizontally laminated sandstone, Sc-convolute laminated sandstone, Sm-massive sandstone, Gh-horizontally stratified gravel, Gt-trough-cross-bedded gravel, CH-channel, LA -lateral-accretion, DA-downstream-accretion, SB-sandy bedform, CS-crevasse splay, FF-floodplain, SL-shallow lake.

\begin{tabular}{|c|c|c|}
\hline Facies association & Description & $\begin{array}{l}\text { Stratigraphic } \\
\text { distribution }\end{array}$ \\
\hline $\begin{array}{l}\text { FA1 } \\
\text { Fine-grained } \\
\text { meandering river } \\
\text { system }\end{array}$ & $\begin{array}{l}\text { Very fine- to medium-grained sandstone and often presence of lateral accretion elements; } \\
\text { floodplain elements are characterized by reddish-brown paleosols with bioturbation, rhizoliths, } \\
\text { desiccation cracks and nodules; relatively high proportion of floodplain elements compared to } \\
\text { channel elements; crevasse splay elements as the proximal part of the floodplain and sandy } \\
\text { bedform deposited as shallow channel fill, channel bars, or point bars deposits are frequently } \\
\text { observed; thickness of sandstone units ranges from } 0.2 \text { to } 7 \mathrm{~m} \text { and mudstone units ranges } \\
\text { from } 0.2 \text { to } 10 \mathrm{~m} \text {. Dominant lithofacies: P, Fl, Fm, Sh, Sr. Minor lithofacies: Sp, St, Sc, Sm. } \\
\text { Architectural elements: FF, CH, LA, CS, SB. }\end{array}$ & $\begin{array}{l}\text { Lower member } \\
\text { and up to middle } \\
\text { part of the middle } \\
\text { member of the } \\
\text { Jagati Formation } \\
(1280 \mathrm{~m})\end{array}$ \\
\hline $\begin{array}{l}\text { FA2 } \\
\text { Flood-flow dominated } \\
\text { meandering river } \\
\text { system }\end{array}$ & $\begin{array}{l}\text { Very fine- to coarse-grained sandstone interbedded with finely laminated greenish-gray } \\
\text { mudstone or reddish brown paleosols; well-developed lateral accretion elements; frequently } \\
\text { observed shallow lake elements characterized by thinly laminated or massive, calcareous } \\
\text { mudstones alternate with thinly bedded, very fine-to fine-grained sandstones with lenticular or } \\
\text { sheet-like geometry; thickness of sandstone units ranges from } 0.2 \text { to } 11 \text { m and mudstone units } \\
\text { ranges from } 0.2 \text { to } 6 \text { m. Dominant lithofacies: P, Fl, Sh, Sm, Fm, Sr, Sp. Minor lithofacies: St, } \\
\text { Sc. Architectural elements: FF, CH, SL, LA, CS, SB. }\end{array}$ & $\begin{array}{l}\text { Upper part of the } \\
\text { middle member } \\
\text { and the upper } \\
\text { member of the } \\
\text { Jagati Formation } \\
(830 \mathrm{~m})\end{array}$ \\
\hline $\begin{array}{l}\text { FA3 } \\
\text { Deep sandy braided } \\
\text { river system }\end{array}$ & $\begin{array}{l}\text { Very fine-to coarse-grained sandstone successions are present in higher proportion than finely } \\
\text { laminated mudstones or reddish-brown paleosols; lateral accretion and downstream accretion } \\
\text { elements are frequently observed; thickness of sandstone units ranges from } 0.2 \text { to } 20 \mathrm{~m} \text { and } \\
\text { mudstone units ranges from } 0.2 \text { to } 5 \mathrm{~m} \text {. Dominant lithofacies: Sh, Sp, St, Sr, P, Fl. Minor } \\
\text { lithofacies: Sm, Sc, Fm. Architectural elements: } \mathrm{CH}, \mathrm{LA}>\mathrm{DA}, \mathrm{SB}, \mathrm{FF} \text {. }\end{array}$ & $\begin{array}{l}\text { Lower and middle } \\
\text { member of the } \\
\text { Kala Formation } \\
(1650 \mathrm{~m})\end{array}$ \\
\hline $\begin{array}{l}\text { FA4 } \\
\text { Shallow sandy braided } \\
\text { river system }\end{array}$ & $\begin{array}{l}\text { Fine-to very coarse-grained sandstone and pebbly sandstone interbedded with finely laminated } \\
\text { greenish-gray, dark-gray, reddish-brown mudstone; relatively high proportion of very thick- } \\
\text { bedded channel elements compared to floodplain elements; downstream-accretion elements } \\
\text { are frequently observed; thickness of sandstone units ranges from } 0.4 \text { to } 21 \mathrm{~m} \text { and mudstone } \\
\text { units ranges from } 0.1 \text { to } 3 \mathrm{~m} \text {. Dominant lithofacies: St, Sp, Gt, Gh, Sh. Minor lithofacies: Fl, } \\
\text { Fm, P, Sr, Sm. Architectural elements: } \mathrm{CH}, \mathrm{DA}>\mathrm{LA}, \mathrm{SB}, \mathrm{FF} \text {. }\end{array}$ & $\begin{array}{l}\text { Upper member } \\
\text { of the Kala } \\
\text { Formation } \\
(400 \mathrm{~m})\end{array}$ \\
\hline
\end{tabular}

\section{METHODOLOGY}

Modal compositions of 67 Siwalik Group sandstone samples from the Khutia Khola section were studied using standard thin-section petrographic analysis and framework modal analysis was quantified using the Gazzi-Dickinson method (Dickinson 1970; Ingersoll et al. 1984; Dickinson 1985). A total of 500 grains were counted per thin section by using a Swift point counter with horizontal grid spacing $0.3 \mathrm{~mm}$ to avoid individual grains being counted more than once. The main categories of grains identified include monocrystalline quartz $(\mathrm{Qm})$, polycrystalline quartz $(\mathrm{Qp})$, plagioclase $(\mathrm{P})$, $\mathrm{K}$-feldspar (K), sedimentary lithic (Ls), carbonate lithic (Lc), metamorphic lithic (Lm), volcanic lithic (Lv) and chert. In addition biotite, muscovite, chlorite, calcite cement, other cements, heavy minerals, altered minerals, accessory minerals, matrix and opaque were also included in point count. The detrital modes were recalculated to $100 \%$ and the parameters were plotted on ternary diagrams, Q-F-L and Q-F-R for classification (Pettijohn 1975; Folk 1980) and, Qt-F-L, QmF-L, Qp-Lv-Ls, Qm-P-K and Lm-Lv-Ls for determination of provenance (Dickinson et al. 1983; Dickinson 1985; Ingersoll and Suczek 1979). Similarly, for determination of climatic indexes bivariant $\log / \log$ diagram of $\mathrm{Qp} / \mathrm{F}+\mathrm{R}$ Vs $\mathrm{Qt} / \mathrm{F}+\mathrm{R}$, bivariant log-ratio diagram of Q/F Vs Q/R (Suttner and Dutta 1986; Weltje et al. 1998) and ternary Q-F-R diagram (Suttner et al. 1981) were plotted. The framework parameters used in this study are listed in Table 2 and recalculated detrital framework compositions (\%) with other calculations are listed in Table 3.

Table 2: Framework parameters used in this study.

\begin{tabular}{|l|l|}
\hline Symbol & Defination \\
\hline Qm & Monocrystalline quartz \\
\hline Qp & Polycrystalline quatz \\
\hline $\mathbf{Q}$ & Total quartz grains $(\mathrm{Qm}+\mathrm{Qp})$ \\
\hline $\mathbf{Q t}$ & Total quartz grains $(\mathrm{Qm}+\mathrm{Qp})+$ chert \\
\hline $\mathbf{P}$ & Plagioclase \\
\hline $\mathbf{K}$ & K-feldspar \\
\hline $\mathbf{F}$ & Total feldspar $(\mathrm{P}+\mathrm{K})$ \\
\hline $\mathbf{L s}$ & Sedimentary lithic fragment \\
\hline $\mathbf{L m}$ & Metamorphic lithic fragment \\
\hline $\mathbf{L v}$ & Volcanic lithic fragment \\
\hline $\mathbf{L}$ & Total lithic fragments $(\mathrm{Ls}+\mathrm{Lm}+\mathrm{Lv})$ \\
\hline $\mathbf{L t}$ & L + Qp + chert \\
\hline $\mathbf{R}$ & Total lithic fragments $(\mathrm{L})+$ chert \\
\hline
\end{tabular}




\section{RESULTS}

\section{Petrography}

\section{Jagati Formation}

Forty-one samples were obtained from the Jagati Formation, seventeen from the lower member, twelve from the middle member and twelve from the upper member were analyzed (Table 3). The Jagati Formation sandstones are mostly matrixpoor, moderately to well-sorted and grains are sub-angular to rounded. Quartz is the dominant detrital constituent (average $57 \%$ ) and monocrystalline quartz is more abundant than polycrystalline quartz (Qm:Qp ratio 65:35) (Fig. 3A). The abundance of quartz is slightly increased from the lower member $(58 \%)$ to the middle member $(60 \%)$ and is decreased in the upper member (55\%). Lithic fragments are the second most abundant detrital constituent overall, and sedimentary (Ls = $56 \%)$ and metamorphic $(\mathrm{Lm}=43 \%)$ lithic fragments are much more abundant than volcanic $(\mathrm{Lv}=1 \%)$ lithic fragments (Fig. $3 \mathrm{C}$ and $\mathrm{D})$. The lithic fragments constituent range from a low $12 \%$ in KS-1 to a high $27 \%$ in KS-30. The sedimentary lithic fragments are mainly shale, siltstone, sandstone, carbonate and chert (Fig. 3B) whereas metamorphic lithic fragments are phyllite and quartz-mica schists and quartzite. Feldspar is the least abundant among QFL and overall range is 2.6 to $8.4 \%$. Feldspars are mainly plagioclase, orthoclase and microcline. The average abundance of feldspare is approximately similar in the lower $(5.5 \%)$ and middle members $(5.0 \%)$ but slightly higher in the upper member (6.4\%). In most samples K-feldspar (K) and plagioclase (P) are approximately subequal, with $\mathrm{P}: \mathrm{K}$ ratio 53:47, 48:52 and 46:54 in the lower, middle and upper members, respectively. Of the total frameworks in the Jagati Formation, mica ranges from 1.8 to $12.2 \%$ (average $5.2 \%$ ) and overall mica contents are $6.2 \%, 3.5 \%$ and $5.6 \%$ in the lower, middle and upper members, respectively. In most samples muscovite (M) content is higher than biotite (B) with an overall $\mathrm{M}: \mathrm{B}$ ratio $57: 43$. Carbonate intraclast is another significant constituent which ranges from 0.4 to $12.6 \%$ in the Jagati Formation. Cement is differentiated into calcite and other cements and overall ratio is 79:21. Trace amounts of a number of heavy minerals also occurs which ranges from 0.2 to $4.2 \%$ (average $1.8 \%$ ), include opaque, zircon, kyanite, sillimanite, garnet, etc.

\section{Kala Formation}

Of the 26 samples were analyzed for the Kala Formation, seventeen were from the lower member, six from the middle member, and three from the upper member (Table 3). The Kala Formation sandstones are poorly to well-sorted, grains are angular to sub-rounded and mostly matrix supported, but grain supported sandstones also occur. Again, quartz is the dominant detrital constituent (average 53\%) with Qm:Qp ratio 64:36. The abundance of quartz increases from the lower member $(51 \%)$ through middle $(55 \%)$ to upper $(57 \%)$ members. Lithic fragments range from 11 to $23 \%$ (average $17 \%$ ), with the average composition of $\mathrm{Ls}=53 \%, \mathrm{Lm}=43 \%$ and $\mathrm{Lv}=4 \%$. The sedimentary lithic fragments constituent decrease up section, whereas metamorphic and volcanic lithic fragments constituent increase up section from lower to upper member. Metamorphic lithic fragments are dominantly quartzmica schist, phyllite and quartzite whereas sedimentary lithic fragments content mudstone, siltstone and carbonate. Feldspar content ranges from 5 to $10 \%$ and is slightly higher in the Kala Formation (average 7.7\%) than in the Jagati Formation (average $5.5 \%$ ). The average constituent of feldspar are approximately similar in all three members (lower $=7.9 \%$, middle $=7.3 \%$ and upper $=8.0 \%$ ). In most samples $\mathrm{K}$-feldspar $(\mathrm{K})$ content is slightly higher than plagioclase $(\mathrm{P})$ with $\mathrm{P}: \mathrm{K}$ ratio 48:52, 48:52 and 50:50 in the lower, middle and upper members, respectively. The amount of mica present ranges from 2.0 to $10.8 \%$ (average 5.8\%) and overall mica contents are 5.5\%, $6.8 \%$ and $5.5 \%$ in the lower, middle and upper members, respectively. Muscovite (M) contents is higher than biotite (B) also in the Kala Formation with an overall M:B ratio 55:45. The average carbonate content is $4.3 \%$ but few samples show higher abundance (up to $14.4 \%, \mathrm{KS}-78$ ). The overall ratio of calcite cement and other cements is 74:26. Heavy minerals and opaque minerals are in minor amount which ranges from 0.6 to $4.6 \%$ but overall content is higher than the Jagati Formation (average 2.9\%).

\section{Sandstone classification}

Major detrital components, quartz, feldspar and lithic fragments, have been recalculated as $100 \%$ for Q-F-L ternary diagrams. Plot in the Q-F-L diagram proposed by Pettijohn (1975) shows that the sandstones are sub-litharenites $(70 \%)$ and lithic arenites (30\%) (Fig. 4 A). Similarly, plot in the Q-F-R diagram proposed by Folk (1980) shows that most of the sandstones are litharenites $(42 \%)$ and feldspathic litharenites (45\%), with fewer sub-litharenites (13\%) (Fig. 4 B). The average modal composition of the Jagati Formation is Q70F7L23 and that of the Kala Formation is Q68F10L22. The overall average composition is Q70F8L22. The Kala sandstones show slightly higher content of feldspar and high quartz/feldspar ratio (average 10.9) is maintained throughout the formation whereas this ratio is slightly less (average 7.0) in the Jagati Formation.

The Khutia Khola sandstone classification (Q-F-R plot) were compared with the Siwalik sections in the Karnali River (Sigdel and Sakai 2013), Surai Khola (Dhital et al. 1995), Arung Khola (Tokuoka et al. 1986) and Samari River (Tamrakar and Syangbo 2014) areas from west towards east in the Nepal Himalaya (Fig. 5). The Karnali River sandstones are classified as litharenite and sub-litharenite, and contain the low amount of feldspar. The Surai Khola and Arung Khola sandstones have the highest amount of feldspar and lithic fragments and are classified as litharenite and feldspathic litharenite, and some as lithic arkose. Similarly, the Samari River sandstones are classified as litharenite through to lithic arkose and contains higher amount of feldspar and quartz than the Khutia Khola sandstones. These Q-F-R diagrams show that the compositions are similar among these Siwalik sections. 

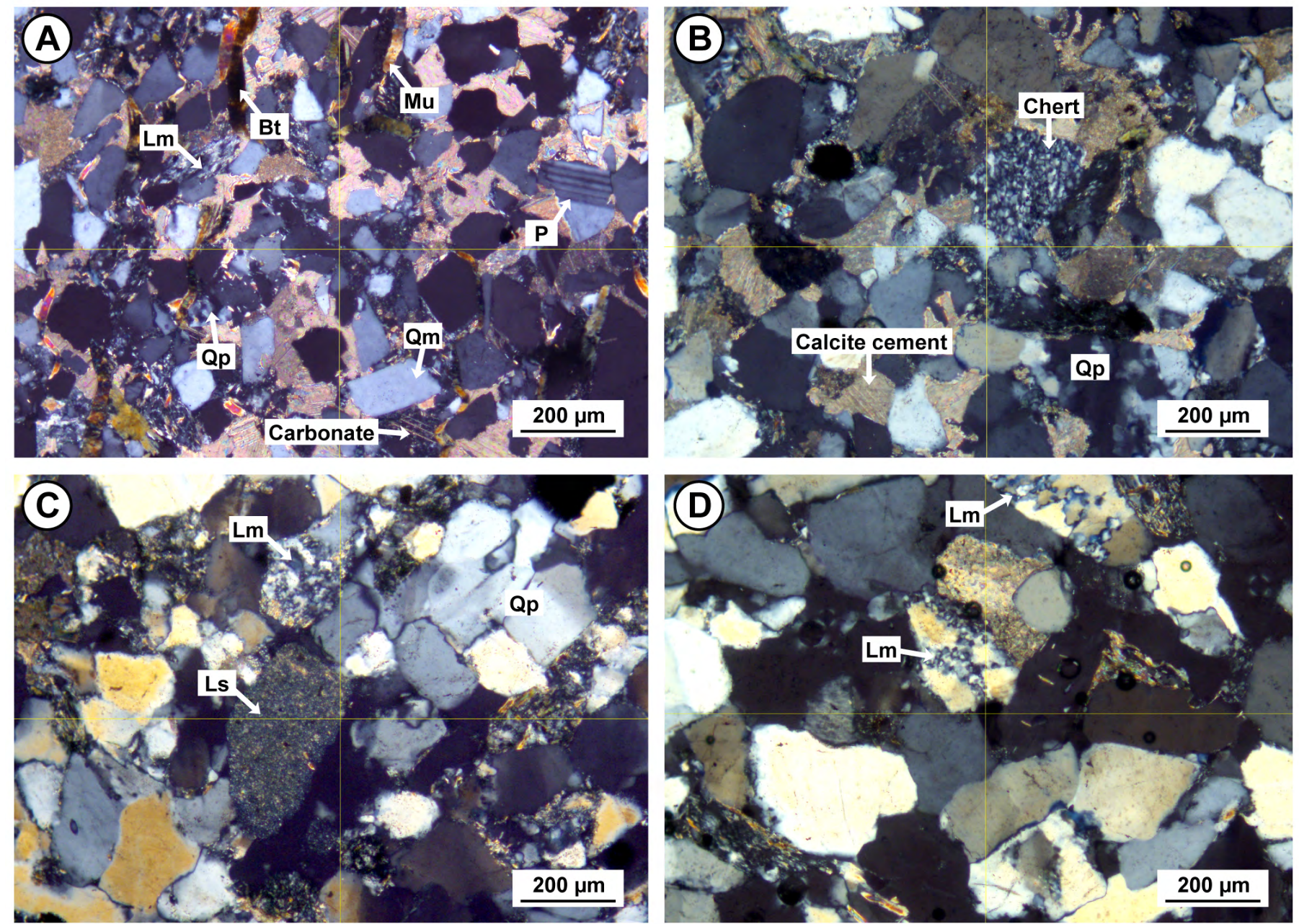

Fig. 3: Photomicrographs of sandstones from the Jagati and Kala Formations. (A) Qp (polycrystalline quartz), Qm (monocrystalline quartz), P (plagioclase), Bt (biotite), Mu (muscovite), Lm (metamorphic lithic fragment), and carbonate. (B) Chert, calcite cement and Qp (polycrystalline quartz). (C) Lm (metamorphic lithic fragment, Ls (sedimentary lithic fragment, and Qp (polycrystalline quartz). (D) Lm (metamorphic lithic fragment).

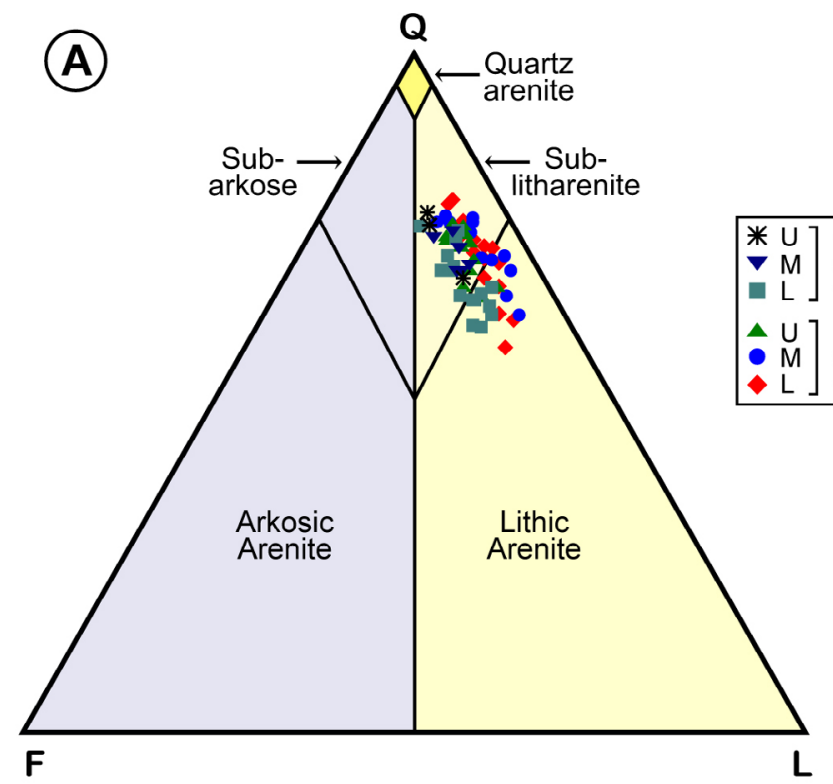

(B)

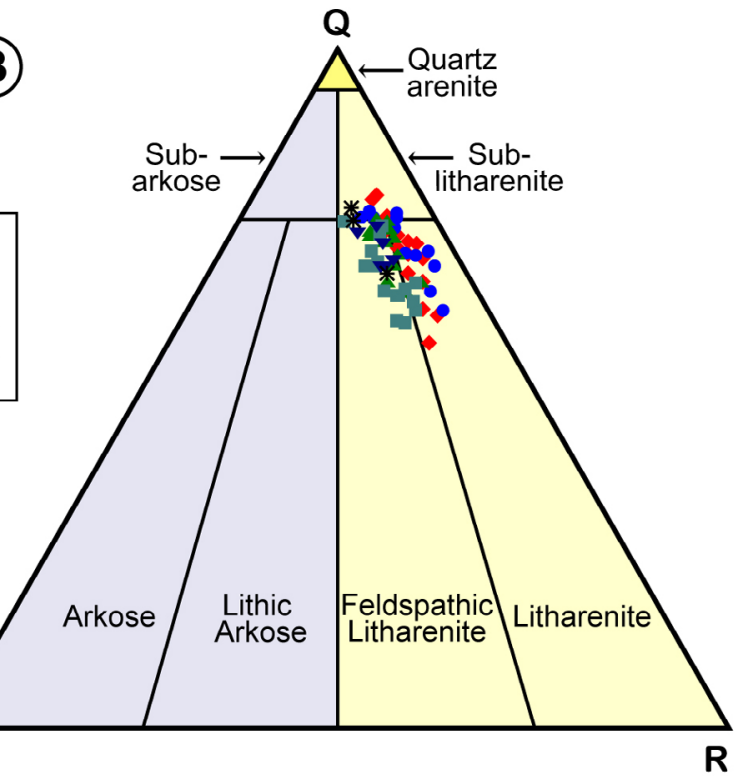

Fig. 4: Ternary diagram for sandstone composition based on schemes proposed by A) Pettijohn (1975); Q-F-L (quart, feldspar, lithic fragments), and B) Folk (1980); Q-F-R (quartz, feldspar, rock fragments). 


\begin{tabular}{|c|c|c|c|c|c|c|c|c|c|c|c|c|c|c|c|c|c|c|c|c|c|c|c|c|c|c|c|c|c|c|c|c|c|}
\hline & & & & & $\stackrel{\substack{i \\
i}}{ }$ & $\begin{array}{ll}3 \\
\end{array}$ & & & $\left|\begin{array}{l}n \\
i\end{array}\right|$ & $\stackrel{\stackrel{i}{i}}{ }$ & הี & $\left|\begin{array}{l}0 \\
i\end{array}\right|$ & $\stackrel{i}{i}$ & & & $\mid \begin{array}{l}6 \\
\end{array}$ & $\underset{i}{\stackrel{0}{i}}$ & $\left|\begin{array}{l}0 \\
\mathrm{i}\end{array}\right|$ & $\vec{i}$ & $\cong$ & 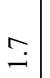 & $\vec{i}$ & $\vec{i}$ & $\exists$ & 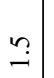 & 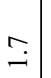 & \begin{tabular}{l|l}
- & \\
- &
\end{tabular} & 9 & $\begin{array}{c}\infty \\
i \\
i\end{array}$ & $\stackrel{\Xi}{-}$ & | & $\vec{i}$ & ? \\
\hline$\stackrel{\Xi}{\stackrel{\Xi}{\Xi}}$ & & & & $\begin{array}{l}0 \\
\stackrel{0}{0}\end{array}$ & $\dot{a}$ & $\exists$ & & : & ò & $\ddot{0}$ & $\hat{o}$ & $\stackrel{0}{-}$ & aे & & : & $\begin{array}{l}0 \\
0 \\
0\end{array}$ & $\hat{o}$ & $\stackrel{\circ}{-}$ & $\begin{array}{l}\infty \\
\vdots \\
\dot{0}\end{array}$ & $\hat{0}$ & $\because$ & $\begin{array}{l}\infty \\
0 \\
0\end{array}$ & $\begin{array}{l}0 \\
0 \\
0\end{array}$ & $\begin{array}{l}0 \\
0 \\
0\end{array}$ & $\stackrel{0}{\circ}$ & : & $\begin{array}{l}0 \\
\circ\end{array}$ & $\stackrel{\infty}{0}$ & $\exists$ & $\stackrel{t}{0}$ & ó & $\hat{o}$ & o \\
\hline & & & & $\exists$ & $\stackrel{0}{-}$ & $\stackrel{9}{-}$ & & $\dot{*}$ & $\stackrel{?}{-}$ & $\exists$ & $\exists$ & $\stackrel{n}{-}$ & $\stackrel{\text { I }}{-}$ & & $\stackrel{\text { I }}{-}$ & $\stackrel{\circ}{-}$ & 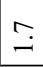 & $\stackrel{m}{-}$ & $\stackrel{m}{\longrightarrow}$ & $\stackrel{\sim}{=}$ & $\grave{0}$ & $\stackrel{\sim}{=}$ & $\stackrel{2}{-}$ & $\grave{\partial}$ & $\begin{array}{l}\infty \\
0 \\
0\end{array}$ & aे & $\tilde{o}$ & $\dot{o}$ & \begin{tabular}{l} 
\pm \\
\hdashline
\end{tabular} & $\tilde{0}$ & 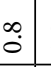 & $\stackrel{\Im}{-}$ & o \\
\hline 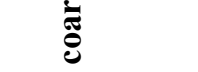 & & $\Xi \partial^{\frac{\pi}{2}}$ & & & $\stackrel{i}{i}$ & $\stackrel{0}{i}$ & & त̃ & $\overrightarrow{\mathrm{i}}$ & 9 & $\vec{i}$ & 9 & 9 & & $\stackrel{\infty}{-}$ & 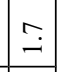 & $\stackrel{\infty}{-}$ & $\begin{array}{l}\tilde{i} \\
\mathrm{i}\end{array}$ & 9 & $\begin{array}{l}\infty \\
- \\
-\end{array}$ & $\stackrel{\circ}{i}$ & 9 & $=$ & $\stackrel{\infty}{-}$ & $\stackrel{\circ}{-}$ & $\stackrel{\infty}{-}$ & $\stackrel{9}{-}$ & $\tilde{N}$ & $\begin{array}{c}m \\
\mathrm{i}\end{array}$ & $\begin{array}{l}0 \\
-\end{array}$ & $g$ & 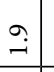 & $\tilde{c}$ \\
\hline & $0^{\circ}$ & 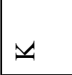 & & î & $\begin{array}{l}n \\
\tilde{y} \\
y\end{array}$ & 咅 & & $\begin{array}{l}y \\
\dot{y} \\
\end{array}$ & $\mid$\begin{tabular}{l}
$n$ \\
\multirow{2}{*}{} \\
$\dot{n}$
\end{tabular} & $\begin{array}{c}0 \\
i \\
i \\
\end{array}$ & $\mid \begin{array}{l}n \\
\vdots \\
\dot{n} \\
i\end{array}$ & $\frac{y}{i n}$ & $\begin{array}{l}n \\
i \\
i\end{array}$ & & $\dot{f}$ & \begin{tabular}{|l|}
$n$ \\
$n$ \\
$n$ \\
$n$
\end{tabular} & $\begin{array}{l}m \\
n \\
n\end{array}$ & $\mid \begin{array}{c}m \\
\tilde{n}\end{array}$ & $\begin{array}{l}\stackrel{0}{\dot{\vec{\sigma}}} \\
\end{array}$ & \begin{tabular}{|c|}
$\infty$ \\
$\infty$ \\
$\infty$ \\
\multirow{1}{*}{} \\
\end{tabular} & $\stackrel{+}{\vec{n}}$ & $\begin{array}{l}\infty \\
\dot{b} \\
\dot{n}\end{array}$ & $\begin{array}{l}f \\
\dot{f}\end{array}$ & $\begin{array}{l}n \\
+ \\
+\end{array}$ & $\begin{array}{l}0 \\
\dot{f}\end{array}$ & $\ddot{n}$ & $\begin{array}{r}m \\
\dot{n}\end{array}$ & $\begin{array}{l}m \\
i \\
i\end{array}$ & $\begin{array}{l}2 \\
i \\
i\end{array}$ & 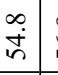 & & & $\begin{array}{l}\sigma \\
\dot{1}\end{array}$ \\
\hline 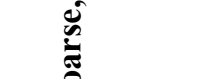 & & $a$ & & $\begin{array}{l}n \\
\stackrel{n}{f} \\
\dot{n}\end{array}$ & $\stackrel{n}{i}$ & $\begin{array}{l}\dot{y} \\
\dot{q}\end{array}$ & & & $\begin{array}{l}n \\
\dot{q} \\
\dot{q}\end{array}$ & $\begin{array}{c}\stackrel{+}{\sim} \\
\vec{f}\end{array}$ & $\begin{array}{l}n \\
n \\
q\end{array}$ & \begin{tabular}{|c|}
$\infty$ \\
$\dot{\alpha}$ \\
$\dot{\alpha}$
\end{tabular} & r & & $\vec{\circ}$ & $\begin{array}{l}n \\
\tilde{y} \\
\tilde{y}\end{array}$ & $\hat{f}$ & $\begin{array}{l}\hat{b} \\
\dot{q}\end{array}$ & $\begin{array}{l}\stackrel{0}{\circ} \\
\dot{n}\end{array}$ & $\stackrel{\text { in }}{\text { in }}$ & $\begin{array}{c}0 \\
\infty \\
\infty \\
+ \\
+\end{array} \mid$ & $\begin{array}{l}\tilde{y} \\
\dot{q} \\
\dot{q}\end{array}$ & $\begin{array}{l}n \\
i \\
i n\end{array}$ & $\begin{array}{l}n \\
i\end{array}$ & $\begin{array}{l}0 \\
0 \\
\dot{b}\end{array}$ & 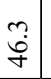 & $\begin{array}{l}\ddot{q} \\
\dot{q}\end{array}$ & $\hat{b}$ & & 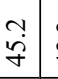 & & 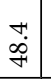 & $\begin{array}{l}0 \\
\text { t }\end{array}$ \\
\hline$=$ & $b^{\circ}$ & $\approx$ & & ָे. & $\stackrel{m}{i}$ & \begin{tabular}{|c|}
$n$ \\
$n$ \\
$n$ \\
\end{tabular} & & & 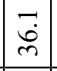 & $\underset{m}{m}$ & $\stackrel{m}{m}$ & $\begin{array}{l}0 \\
\dot{2} \\
\dot{m}\end{array}$ & $\begin{array}{l}n \\
? \\
+ \\
\end{array}$ & & & $\vec{i}$ & $\begin{array}{l}n \\
\infty \\
\infty \\
\sim \\
\end{array}$ & $\begin{array}{l}n \\
\dot{q} \\
\end{array}$ & $\stackrel{\sim}{m}$ & \begin{tabular}{|l|} 
\\
$\dot{y}$ \\
\end{tabular} & $\begin{array}{l}0 \\
\dot{i}\end{array}$ & $\begin{array}{l}\infty \\
\dot{m} \\
\dot{m}\end{array}$ & \begin{tabular}{|l|} 
\\
$\dot{e}$ \\
\end{tabular} & $\begin{array}{c}m \\
m \\
m\end{array}$ & $\begin{array}{l}0 \\
\dot{y} \\
\text {. }\end{array}$ & $\dot{m}$ & $\begin{array}{l}0 \\
\dot{D} \\
\dot{m}\end{array}$ & $\begin{array}{l}m \\
\vec{\nabla}\end{array}$ & & \begin{tabular}{l|l}
$\infty$ \\
$\dot{0}$ \\
$\dot{\sim}$
\end{tabular} & & $\begin{array}{l}0 \\
\dot{\rho} \\
\dot{m}\end{array}$ & in \\
\hline & & $\Xi$ & & $\stackrel{m}{i}$ & $\stackrel{\hat{\sigma}}{\hat{\sigma}}$ & 旾 & & & $\overrightarrow{8}$ & 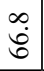 & 今. & $\begin{array}{l}+ \\
\dot{B}\end{array}$ & $\ddot{s}$ & & i & iे & $\stackrel{n}{r}$ & $\left|\begin{array}{l}\infty \\
\vdots \\
\dot{n}\end{array}\right|$ & $\begin{array}{l}\infty \\
\dot{0} \\
\dot{0}\end{array}$ & $\mid \begin{array}{r}\vec{d} \\
\vec{i}\end{array}$ & $\stackrel{+}{\stackrel{2}{R}}$ & है & $\left|\begin{array}{l}0 \\
0 \\
0\end{array}\right|$ & \begin{tabular}{l}
$\mid$ \\
0 \\
\hdashline \\
0
\end{tabular} & $\begin{array}{l}0 \\
\dot{\infty} \\
i\end{array}$ & तु & $\begin{array}{l}\dot{8} \\
\dot{6}\end{array}$ & $\stackrel{r}{\infty}$ & & $\begin{array}{l}\tilde{c} \\
\tilde{n}\end{array}$ & & ț & is \\
\hline & $\partial^{\circ}$ & 3 & & $\begin{array}{l}\infty \\
\dot{m} \\
\dot{m}\end{array}$ & $\hat{\sigma}$ & 它 & & & $\hat{\vec{i}}$ & 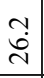 & हे & $\mid \begin{array}{l}\stackrel{ \pm}{ \pm} \\
\end{array}$ & : & & & $\begin{array}{l}\infty \\
\dot{m} \\
\end{array}$ & ন্ & $\mid \begin{array}{l}\infty \\
\ddot{i} \\
\end{array}$ & $\begin{array}{l}\infty \\
\dot{m} \\
\end{array}$ & $\mid \begin{array}{l}n \\
i \\
i\end{array}$ & $\begin{array}{l}0 \\
\dot{\sigma} \\
\dot{f}\end{array}$ & $\vec{m}$ & $\begin{array}{l}\vec{j} \\
\dot{m}\end{array}$ & $\begin{array}{l}0 \\
\text { ले } \\
m\end{array}$ & $\stackrel{a}{\dot{m}}$ & $\vec{m}$ & $\begin{array}{l}\tilde{m} \\
\end{array}$ & $\begin{array}{c}0 \\
\dot{q} \\
\dot{q}\end{array}$ & & $\begin{array}{l}r \\
\dot{a}\end{array}$ & & $\frac{n}{m}$ & 3 \\
\hline & & 3 & & 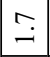 & $\stackrel{0}{i}$ & $\hat{i}$ & & 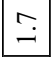 & $\hat{0}$ & $\stackrel{m}{m}$ & Iี & $\stackrel{m}{\stackrel{m}{+}}$ & $\stackrel{n}{+}$ & & $\vec{i}$ & $\because$ & 9 & $\stackrel{\dot{m}}{\stackrel{\sim}{*}}$ & $\stackrel{0}{-}$ & $\because$ & $\stackrel{\circ}{i}$ & $\stackrel{0}{\circ}$ & \begin{tabular}{|l|}
$\infty$ \\
0 \\
0
\end{tabular} & $\tilde{n}$ & $\vec{i}$ & $\stackrel{\nabla}{\sim}$ & $\vec{i}$ & $\stackrel{0}{\circ}$ & $\stackrel{\circ}{\circ}$ & $\stackrel{\circ}{\circ}$ & 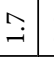 & İ & 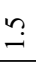 \\
\hline & & $\approx$ & & 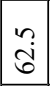 & $\hat{\dot{\infty}}$ & $\overrightarrow{\dot{8}}$ & & & $\hat{\mathrm{g}}$ & 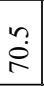 & ठें & $\begin{array}{c}m \\
\infty \\
\infty\end{array}$ & शे & & $\frac{m}{6}$ & $\begin{array}{l}\tilde{y} \\
\ddot{b}\end{array}$ & in & $\begin{array}{l}\infty \\
i \\
i\end{array}$ & $\widehat{\hat{b}}$ & \begin{tabular}{|l|}
$\infty$ \\
$\dot{I}$ \\
\end{tabular} & $\begin{array}{l}m \\
\vdots \\
i\end{array}$ & fे & \begin{tabular}{|l|}
$\infty$ \\
$\dot{j}$ \\
$\dot{b}$
\end{tabular} & तై & กั่ & \begin{tabular}{l}
$n$ \\
\hdashline \\
0 \\
0
\end{tabular} & 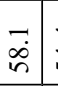 & $\stackrel{+}{\stackrel{H}{*}}$ & & \begin{tabular}{l|l}
$\infty$ \\
0 \\
$\vdots$ \\
$\vdots$ \\
\end{tabular} & & 䓒 & $\therefore$ \\
\hline & $\delta^{\circ}$ & t & & $\stackrel{m}{i}$ & $\stackrel{+}{n}$ & 紊 & & & 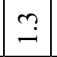 & $\begin{array}{l}\infty \\
+ \\
+\end{array}$ & $\stackrel{+}{\oplus}$ & $\hat{a}$ & $\begin{array}{l}n \\
\infty \\
\infty\end{array}$ & & F & $\ddot{0}$ & $\stackrel{\infty}{\dot{m}}$ & $\stackrel{2}{i}$ & $\begin{array}{l}\infty \\
i \\
i\end{array}$ & $\stackrel{\circ}{\circ}$ & $\ddot{\dot{m}}$ & $\stackrel{0}{\circ}$ & $\stackrel{?}{-}$ & b. & $\stackrel{n}{+}$ & $\vec{n}$ & $\begin{array}{l}\dot{0} \\
\dot{m}\end{array}$ & $\stackrel{0}{0}$ & $\stackrel{0}{0}$ & $\stackrel{r}{+}$ & $\mathrm{i}$ & 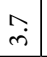 & $\bar{i}$ \\
\hline & $\bar{\Xi}$ & $\Xi$ & & $\begin{array}{l}\stackrel{\sigma}{o} \\
\dot{\alpha}\end{array}$ & $\hat{8}$ & in & & & $\begin{array}{l}\dot{\rho} \\
\dot{\gamma}\end{array}$ & 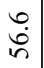 & $\underset{\dot{\sigma}}{\stackrel{+}{*}}$ & $\mid \begin{array}{c}\overrightarrow{0} \\
i n\end{array}$ & $\stackrel{\infty}{\stackrel{\infty}{n}}$ & & $\frac{F}{y}$ & $\begin{array}{l}\hat{\sigma} \\
\dot{\sigma}\end{array}$ & in & 户े & $\begin{array}{l}m \\
\dot{a} \\
\end{array}$ & $\vec{n}$ & $\stackrel{\vec{\sim}}{\stackrel{\sim}{*}}$ & $\begin{array}{l}\infty \\
\grave{i} \\
\grave{i}\end{array}$ & $\mid \vec{i}$ & $\begin{array}{l}0 \\
\dot{n}\end{array}$ & 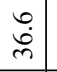 & İ & $\bar{f}$ & 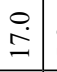 & $\begin{array}{c}\dot{n} \\
\dot{n}\end{array}$ & $\begin{array}{l}\infty \\
\infty \\
\dot{\infty} \\
\dot{e}\end{array}$ & g & $\begin{array}{l}n \\
\tilde{y} \\
y \\
y\end{array}$ & I \\
\hline 8 & & 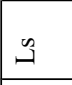 & & $\begin{array}{c}\dot{a} \\
\dot{\alpha} \\
\end{array}$ & ले & \begin{tabular}{|l} 
\\
$\dot{q}$ \\
$\dot{q}$
\end{tabular} & & & $\begin{array}{l}\overrightarrow{8} \\
\dot{n}\end{array}$ & $\begin{array}{l}0 \\
\dot{\infty} \\
\dot{m} \\
\end{array}$ & $\begin{array}{l}\stackrel{y}{y} \\
\stackrel{y}{y}\end{array}$ & 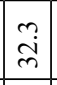 & $\frac{\vec{m}}{m}$ & & $\begin{array}{l}\infty \\
\dot{n} \\
\dot{n}\end{array}$ & $\begin{array}{l}m \\
i \\
i n\end{array}$ & \begin{tabular}{|l} 
\\
s. \\
$b$ \\
\end{tabular} & $\begin{array}{c}m \\
\dot{q} \\
\end{array}$ & $\begin{array}{l}\text { iे. } \\
\text { in }\end{array}$ & \begin{tabular}{|l|}
$\hat{y}$ \\
$\dot{y}$ \\
\end{tabular} & $\begin{array}{l}n \\
8\end{array}$ & $\stackrel{2}{2}$ & $\begin{array}{l}\mid \begin{array}{l}\mid \\
\dot{0} \\
i\end{array} \\
\end{array}$ & $\vec{\sigma}$ & $\begin{array}{l}a \\
\infty \\
n \\
n\end{array}$ & & $\begin{array}{c}m \\
i \\
i\end{array}$ & $\begin{array}{l}0 \\
\dot{\infty} \\
\infty\end{array}$ & & $\begin{array}{l}n \\
\infty \\
\infty \\
n\end{array}$ & & $\begin{array}{c}\hat{i} \\
\text { in }\end{array}$ & $g$ \\
\hline & & & & $\stackrel{2}{2}$ & $\stackrel{?}{n}$ & กั & & & $\mid \begin{array}{l}n \\
\infty \\
\infty\end{array}$ & $\because$ & $\underset{\infty}{+\infty}$ & 星 & $\stackrel{9}{=}$ & & $m$ & $\grave{\grave{I}}$ & $\hat{a}$ & $\mid \begin{array}{c}n \\
\infty \\
\infty\end{array}$ & 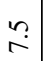 & $\mid \begin{array}{l}\infty \\
\stackrel{0}{-}\end{array}$ & $\begin{array}{l}n \\
\infty \\
\infty\end{array}$ & $\exists$ & $a$ & ạ & $\overrightarrow{ \pm}$ & $\stackrel{m}{=}$ & $\stackrel{m}{\varrho}$ & $\begin{array}{l}n \\
\infty \\
\infty\end{array}$ & $\stackrel{9}{\stackrel{9}{9}}$ & $\stackrel{m}{=}$ & $z$ & $\hat{a}$ & $\infty$ \\
\hline & 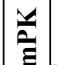 & & & $\underset{\infty}{\dot{\infty}}$ & 2 & $\begin{array}{l}b \\
\end{array}$ & & & $\vec{\therefore}$ & $\begin{array}{l}n \\
\infty \\
\infty\end{array}$ & $\stackrel{\circ}{\stackrel{2}{r}}$ & $\left|\begin{array}{c}\infty \\
a \\
\sigma\end{array}\right|$ & $\hat{\varrho}$ & & $\stackrel{0}{\circ}$ & $\dot{a}$ & $\mid \begin{array}{l}n \\
\infty \\
\infty\end{array}$ & $\underset{r}{\stackrel{\sim}{r}}$ & $\begin{array}{l}\infty \\
\stackrel{\infty}{\varrho}\end{array}$ & $\stackrel{\stackrel{?}{=}}{=}$ & $\vec{\infty}$ & ֵֵ) & $\stackrel{\ominus}{=}$ & $\overrightarrow{0}$ & 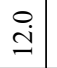 & $\ddot{a}$ & $\begin{array}{c}\hat{\infty} \\
\end{array}$ & $\stackrel{+}{\stackrel{\sim}{r}}$ & \begin{tabular}{l|l}
$\infty$ \\
$\dot{n}$ \\
$\dot{n}$ & 0
\end{tabular} & $\stackrel{t}{\sigma}$ & $\infty$ & $\vec{a}$ & 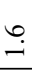 \\
\hline & & $\Xi$ & & $\begin{array}{l}m \\
i \\
\infty\end{array}$ & $\begin{array}{l}\infty \\
\infty \\
\infty \\
\infty\end{array}$ & $\underset{\infty}{\infty}$ & & & $\mid \begin{array}{l}+ \\
\dot{\infty}\end{array}$ & $\begin{array}{c}0 \\
i \\
\infty\end{array}$ & $\stackrel{\text { f }}{\dot{+}}$ & $\begin{array}{l}0 \\
\dot{\infty}\end{array}$ & i & & $\vec{\infty}$ & $\mid \begin{array}{l}1 \\
\stackrel{2}{1}\end{array}$ & $\vec{\infty}$ & $\overrightarrow{\dot{D}}$ & $\begin{array}{l}\stackrel{0}{\infty} \\
\dot{\infty}\end{array}$ & $\mid \begin{array}{l}\stackrel{a}{i} \\
\stackrel{i}{R}\end{array}$ & $\underset{\infty}{\dot{\infty}}$ & $\hat{i}$ & $\mid \begin{array}{c}7 \\
2 \\
2\end{array}$ & $\hat{\infty}$ & $\begin{array}{l}\mid \\
\stackrel{+}{+}\end{array}$ & $\therefore$ & $\frac{0}{\dot{\infty}}$ & $\begin{array}{l}0 \\
\dot{\Phi}\end{array}$ & \begin{tabular}{l|l}
$\tilde{B}$ & \\
$\infty$ & \\
$\infty$
\end{tabular} & $\frac{m}{2}$ & & 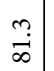 & $\stackrel{M}{M}$ \\
\hline & & & & & $m$ & 享 & & & $\left|\begin{array}{l}1 \\
\text { gु }\end{array}\right|$ & $\begin{array}{c}m \\
\dot{q} \\
\dot{q}\end{array}$ & fo & $\mid \begin{array}{c}\dot{y} \\
\dot{g}\end{array}$ & $\overrightarrow{i n}$ & & & 苍 & & $\left|\begin{array}{l}\infty \\
\dot{\delta} \\
\dot{q}\end{array}\right|$ & $\begin{array}{l}\vec{j} \\
\vec{q}\end{array}$ & & $\begin{array}{l}0 \\
\dot{f}\end{array}$ & $\begin{array}{l}\dot{a} \\
\dot{q}\end{array}$ & & $\begin{array}{l}\dot{0} \\
\dot{\phi} \dot{\sigma}\end{array}$ & $\hat{n}$ & & 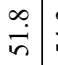 & $\begin{array}{l}m \\
\stackrel{m}{n}\end{array}$ & \begin{tabular}{l|l}
$\mathfrak{y}$ & \multirow{y}{*}{} \\
\cline { 1 - 1 }
\end{tabular} & 字 & & 아 & in \\
\hline & 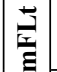 & I & & $\overrightarrow{0}$ & $\stackrel{+}{\ominus}$ & 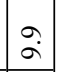 & & & \begin{tabular}{|l|}
+ \\
$\infty$ \\
$\infty$
\end{tabular} & 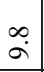 & $n_{\infty}^{\infty}$ & 产 & $\stackrel{\overbrace{}}{\varrho}$ & & $\stackrel{\sim}{=}$ & $\stackrel{?}{=}$ & $\stackrel{+}{\exists}$ & $\vec{\infty}$ & $\begin{array}{l}n \\
\varrho \\
0\end{array}$ & 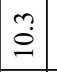 & $\stackrel{\partial}{\infty}$ & $\stackrel{?}{0}$ & 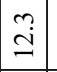 & $\overrightarrow{0}$ & $\stackrel{\mathfrak{I}}{\Xi}$ & $\begin{array}{l}0 \\
\stackrel{0}{-1}\end{array}$ & 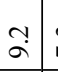 & $\stackrel{?}{?}$ & $\stackrel{?}{?}$ & $\stackrel{I}{\Xi}$ & & $\stackrel{\sigma}{2}$ & 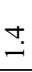 \\
\hline & & $\Xi$ & & & in & $\vec{g}$ & & & $\mid \begin{array}{c}\dot{y} \\
\dot{y}\end{array}$ & $\begin{array}{l}\infty \\
\dot{j} \\
\dot{f}\end{array}$ & $\vec{b}$ & $\mid \begin{array}{l}\overrightarrow{\dot{g}} \\
\end{array}$ & $\begin{array}{l}\infty \\
\dot{m}\end{array}$ & & & $\left|\begin{array}{c}m \\
\infty \\
\infty\end{array}\right|$ & in & $\overrightarrow{\vec{g}}$ & $\begin{array}{l}0 \\
\dot{\sigma} \\
\dot{q}\end{array}$ & 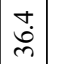 & 守 & $\begin{array}{l}0 \\
\dot{+} \\
\dot{q}\end{array}$ & $\begin{array}{c}\stackrel{?}{f} \\
f\end{array}$ & $\begin{array}{l}\stackrel{i}{\mathrm{i}} \\
\vec{\gamma}\end{array}$ & $\underset{\dot{m}}{\stackrel{f}{m}}$ & $m$ & $\begin{array}{l}\circ \\
\dot{m}\end{array}$ & 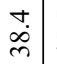 & 兮 & $\stackrel{0}{\dot{q}}$ & & $\underset{q}{q}$ & is \\
\hline & & & & $\begin{array}{l}0 \\
\dot{\lambda} \\
\text {. }\end{array}$ & $\dot{I}$ & $\begin{array}{l}n \\
m \\
m\end{array}$ & & & $\stackrel{a}{\partial}$ & $\begin{array}{l}\infty \\
\dot{\vec{v}}\end{array}$ & $\begin{array}{l}n \\
i \\
i\end{array}$ & in & $\stackrel{+}{\vec{\sim}}$ & & $\stackrel{+}{\stackrel{i}{े}}$ & $\begin{array}{l}\infty \\
\dot{\lambda} \\
\end{array}$ & $\dot{\vec{g}}$ & $\stackrel{m}{2}$ & $\stackrel{n}{2}$ & $\mid \begin{array}{l}\infty \\
\stackrel{i}{i} \\
\end{array}$ & ֶֶّ & $\stackrel{\circ}{\dot{\vec{N}}}$ & $\begin{array}{l}0 \\
\vdots \\
=\end{array}$ & : & $\begin{array}{l}n \\
\stackrel{n}{v}\end{array}$ & त1 & $\overrightarrow{\mathrm{i}}$ & $\stackrel{?}{\stackrel{n}{\sim}}$ & $\stackrel{\circ}{\stackrel{9}{9}}:$ & $\begin{array}{l}\infty \\
\dot{d} \\
\dot{\sim}\end{array}$ & & $\stackrel{\infty}{\dot{i}}$ & $\underset{7}{f}$ \\
\hline & oे & & & $\stackrel{m}{\varrho}$ & $\underline{n}$ & $\stackrel{\circ}{\stackrel{0}{0}}$ & & & \begin{tabular}{|l|}
+ \\
$\infty$ \\
$\infty$
\end{tabular} & $\stackrel{\circ}{\stackrel{\ominus}{9}}$ & $\underset{\infty}{+}$ & $\begin{array}{l}0 \\
= \\
\end{array}$ & $\stackrel{+}{\stackrel{\Xi}{\varrho}}$ & & $\stackrel{n}{=}$ & $\stackrel{9}{\Xi}$ & $\stackrel{\infty}{\stackrel{\infty}{=}}$ & \begin{tabular}{|c|}
\multirow{2}{*}{} \\
$\infty$
\end{tabular} & $\begin{array}{l}n \\
\varrho \\
\varrho\end{array}$ & $\exists$ & $\vec{a}$ & 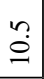 & $\stackrel{\stackrel{\Omega}{I}}{\mathrm{I}}$ & \begin{tabular}{l}
1 \\
\hdashline
\end{tabular} & 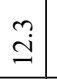 & 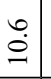 & ָૂ. & $\stackrel{?}{?}$ & $\stackrel{?}{?}=$ & $\stackrel{\Xi}{=}$ & & $\begin{array}{l}0 \\
\stackrel{1}{9}\end{array}$ & 3 \\
\hline & & 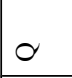 & & $\overrightarrow{5}$ & & $\begin{array}{l}0 \\
\dot{0} \\
\stackrel{2}{2}\end{array}$ & & & $\mid \begin{array}{l}0 \\
\dot{R}\end{array}$ & 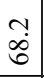 & $\overrightarrow{8}$ & $\vec{m}$ & $\overrightarrow{0}$ & & \begin{tabular}{c}
\multirow{1}{*}{} \\
$\infty$ \\
$\infty$
\end{tabular} & \begin{tabular}{l}
$\mathcal{Y}$ \\
\multirow{H}{*}{} \\
\end{tabular} & $\stackrel{\overbrace{}}{+}$ & $\left|\begin{array}{l}n \\
i \\
i\end{array}\right|$ & $\stackrel{\circ}{\circ}$ & $\vec{\infty}$ & $\begin{array}{l}\infty \\
\dot{J} \\
\end{array}$ & $\begin{array}{l}n \\
0 \\
0 \\
0\end{array}$ & $\overrightarrow{|c|}$ & ते & $\begin{array}{l}0 \\
\tilde{B}\end{array}$ & శై & $\overrightarrow{\dot{b}}$ & $\begin{array}{l}n \\
\vdots \\
0\end{array}$ & $\stackrel{8}{i}$ & $\begin{array}{l}\infty \\
i \\
i\end{array}$ & & $\begin{array}{c}n \\
\infty \\
\infty \\
0 \\
0\end{array}$ & 7 \\
\hline 8 & & & & $\begin{array}{l}\tilde{N} \\
\text { ה }\end{array}$ & \pm & $\stackrel{+}{\ddot{\theta}}$ & & $\stackrel{2}{\Xi}$ & $\begin{array}{l}\infty \\
\stackrel{2}{2}\end{array}$ & $\frac{n}{\dot{v}}$ & さ্ & ì & $\begin{array}{l}\infty \\
\stackrel{i}{0} \\
\stackrel{i}{1}\end{array}$ & & $\vec{v}$ & $\begin{array}{l}0 \\
\text { i } \\
\end{array}$ & $\stackrel{\forall}{\ddot{m}}$ & $\begin{array}{l}1 \\
2 \\
2\end{array}$ & $\stackrel{\nabla}{\stackrel{2}{二}}$ & $\stackrel{\Delta}{\stackrel{2}{2}}$ & $\begin{array}{l}0 \\
\dot{a} \\
i\end{array}$ & $\hat{\stackrel{i}{े}}$ & $\begin{array}{l}0 \\
\vdots \\
2\end{array}$ & 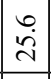 & $\begin{array}{l}\stackrel{+}{\sim} \\
\stackrel{\sim}{v}\end{array}$ & $\ddot{i}$ & $\overrightarrow{\mathrm{i}}$ & $\stackrel{m}{i}$ & \begin{tabular}{l}
$\stackrel{9}{\square}$ \\
\hdashline
\end{tabular} & 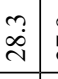 & & 年 & $\begin{array}{l}0 \\
7 \\
1\end{array}$ \\
\hline & & w & & $\overrightarrow{0}$ & $\stackrel{+}{\stackrel{\theta}{\circ}}$ & 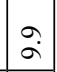 & & $\vec{\infty}$ & \begin{tabular}{|l|}
+ \\
$\infty$ \\
$\infty$
\end{tabular} & $\stackrel{\infty}{a}$ & $\begin{array}{c}n \\
\infty \\
\infty\end{array}$ & $\stackrel{\varrho}{0}$ & $\stackrel{\overbrace{}}{\varrho}$ & & $\stackrel{\overbrace{}}{=}$ & $\stackrel{?}{=}$ & $\stackrel{+}{\Xi}$ & $\vec{\infty}$ & $\begin{array}{l}n \\
\varrho \\
0\end{array}$ & $\stackrel{m}{\varrho}$ & $\stackrel{\infty}{\infty}$ & $\stackrel{m}{\varrho}$ & $\therefore$ & $\overrightarrow{0}$ & $\stackrel{I}{I}$ & $\stackrel{\circ}{\stackrel{0}{\ominus}}$ & ָุa & $\stackrel{?}{r}$ & $\stackrel{?}{?}$ & $\stackrel{\overbrace{}}{=}$ & $\infty$ & $\dot{\alpha}$ & 5 \\
\hline 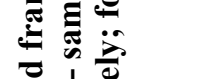 & & 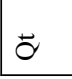 & & i & $\overrightarrow{i n}$ & ڤ. & & 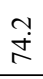 & $\begin{array}{l}\infty \\
\stackrel{i}{r}\end{array}$ & 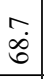 & a. & 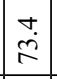 & :̊. & & & $\overrightarrow{\dot{b}}$ & هُ & $\left|\begin{array}{l}6 \\
i \\
i\end{array}\right|$ & $\overrightarrow{\dot{\varepsilon}}$ & $\stackrel{m}{2}$ & $\begin{array}{l}0 \\
\dot{b}\end{array}$ & $\begin{array}{l}0 \\
\dot{8}\end{array}$ & $\begin{array}{c}-1 \\
0 \\
0\end{array}$ & $\mid \begin{array}{l}m \\
\dot{b}\end{array}$ & $\begin{array}{l}+ \\
\dot{0}\end{array}$ & 6 & $\begin{array}{l}\infty \\
\overrightarrow{0}\end{array}$ & $\begin{array}{l}n \\
3 \\
6\end{array}$ & 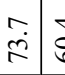 & $\begin{array}{l} \pm \\
\dot{0}\end{array}$ & & $\begin{array}{l}0 \\
\infty \\
0\end{array}$ & 7 \\
\hline 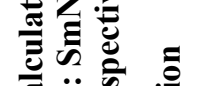 & $\Xi$ & & & $\tilde{\theta}$ & & $\tilde{0}$ & & $\tilde{0}$ & 0 & 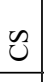 & 0 & $\tilde{0}$ & $>$ & & $\tilde{0}$ & $\tilde{0}$ & 5 & $\tilde{0}$ & I & $\begin{array}{l}0 \\
0 \\
>\end{array}$ & 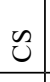 & $\begin{array}{l}\tilde{D} \\
>\end{array}$ & $\sum^{\infty}$ & $\tilde{0}$ & 0 & $\sum^{\infty}$ & 江 & 0 & $\begin{array}{lll}5 \\
\end{array}$ & $\sum^{n}$ & U & & \\
\hline 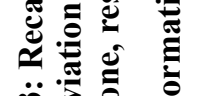 & 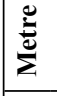 & & $\sum_{i=1}^{n}$ & + & 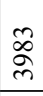 & $\mid \begin{array}{l}\infty \\
\infty \\
\infty \\
\infty\end{array}$ & & & \begin{tabular}{|c|}
0 \\
ర్ల \\
\end{tabular} & 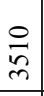 & $\stackrel{\vec{N}}{\vec{n}}$ & $\frac{\approx}{m}$ & $\begin{array}{l}\text { ¿े } \\
\text { ì }\end{array}$ & & $\begin{array}{l}\stackrel{2}{\infty} \\
\text { ì }\end{array}$ & $\begin{array}{l}0 \\
\infty \\
\infty \\
\sim\end{array}$ & 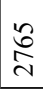 & $\begin{array}{l}\stackrel{\mathrm{N}}{\mathrm{i}} \\
\end{array}$ & $\underset{\vec{N}}{\vec{N}}$ & $\mid \begin{array}{l}0 \\
0 \\
0 \\
ن\end{array}$ & 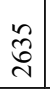 & $\begin{array}{l}n \\
\infty \\
i \\
\end{array}$ & $\begin{array}{l}0 \\
0 \\
i \\
\end{array}$ & $\begin{array}{c}\tilde{T} \\
\underset{\sim}{\sim}\end{array}$ & 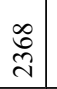 & $\widetilde{\imath}$ & 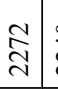 & 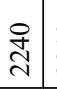 & 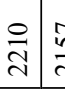 & $\frac{\hat{n}}{\sim}$ & & & \\
\hline$\frac{\pi}{\pi}$ & $\mathrm{Z}$ & & & & & 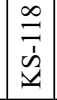 & & $\begin{array}{l}0 \\
\ddots \\
\dot{v} \\
\underline{y}\end{array}$ & & $\tilde{\varkappa}$ & & & $\begin{array}{l}\stackrel{8}{1} \\
\dot{\omega} \\
\underline{y}\end{array}$ & & 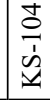 & $\mid \begin{array}{l}\tilde{0} \\
\dot{1} \\
\dot{\hat{\nu}}\end{array}$ & & $\mid \begin{array}{l}\infty \\
\dot{1} \\
\hat{v} \\
\imath\end{array}$ & $\begin{array}{l}\hat{a} \\
\hat{\hat{\nu}} \\
\underline{\Delta}\end{array}$ & $\mid$\begin{tabular}{l} 
\pm \\
$\dot{1}$ \\
$\hat{v}$ \\
\cline { 1 - 1 }
\end{tabular} & $\begin{array}{l}\dot{v} \\
\dot{y}\end{array}$ & $\begin{array}{l}\overline{\widehat{a}} \\
\hat{v} \\
\underline{v}\end{array}$ & $\left|\begin{array}{l}0 \\
\vdots \\
0 \\
\right.$\cline { 1 - 1 }\end{array}$|$ & 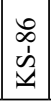 & 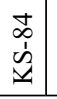 & & \begin{tabular}{l}
8 \\
0 \\
$\vdots$ \\
$\tilde{n}$ \\
\cline { 1 - 1 }
\end{tabular} & $\begin{array}{l}\infty \\
\hat{1} \\
\hat{v} \\
\underline{y}\end{array}$ & 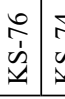 & 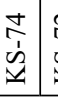 & & $\sum_{\bar{E}}^{\bar{\varpi}}$ & \\
\hline
\end{tabular}




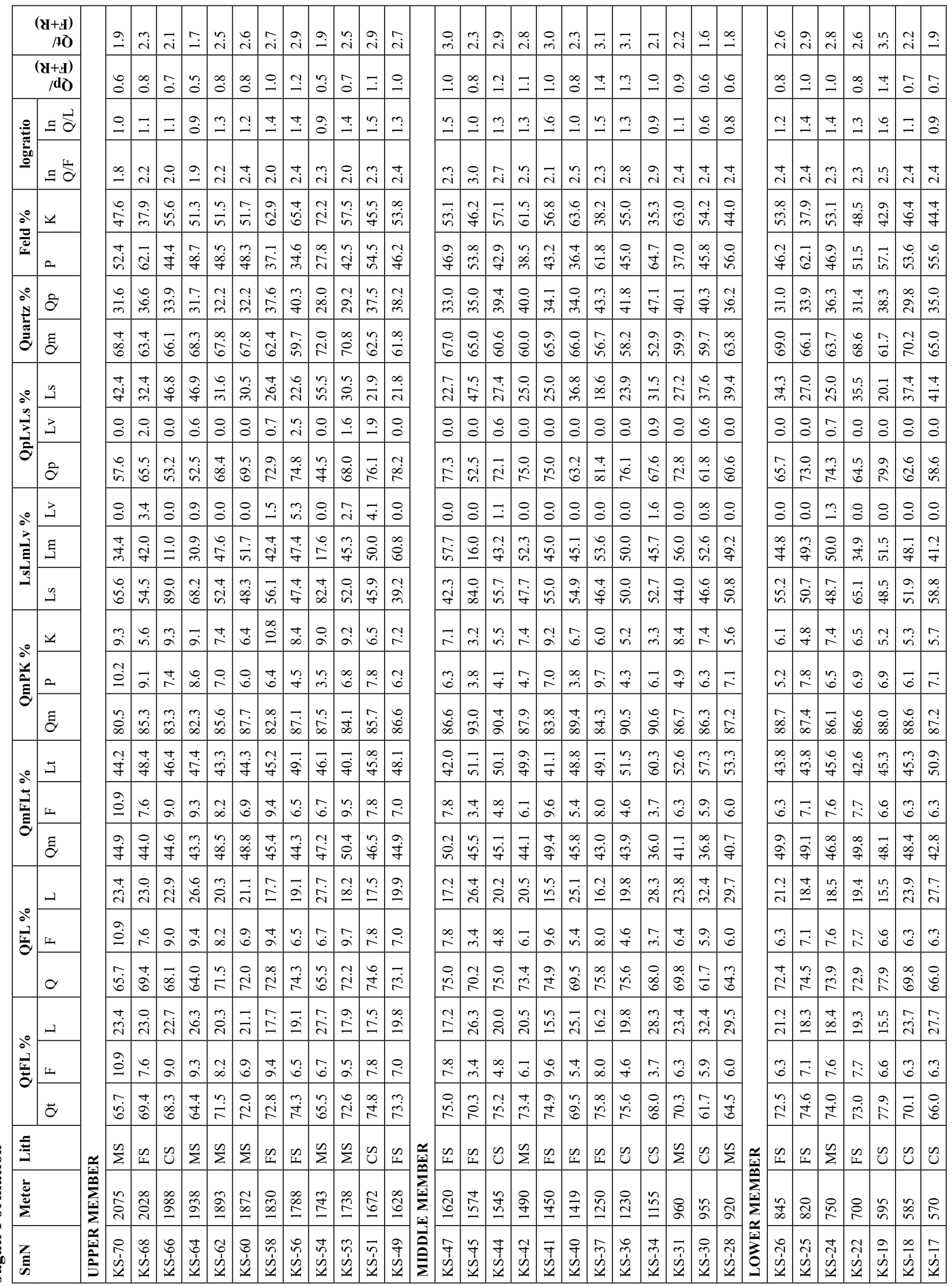




\begin{tabular}{|c|c|c|c|c|c|c|c|c|c|c|c|c|c|c|}
\hline \multirow{6}{*}{\multicolumn{2}{|c|}{ 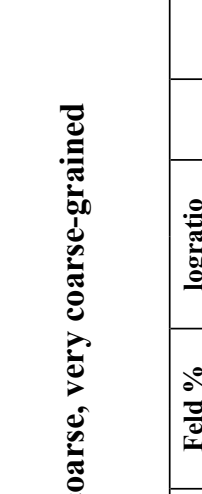 }} & \multirow{2}{*}{ 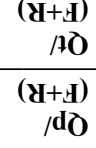 } & \multirow{2}{*}{\begin{tabular}{|c|} 
\\
\hdashline \\
\\
\\
\end{tabular}} & \multirow[b]{2}{*}{$\because$} & \multirow{2}{*}{\multicolumn{2}{|c|}{ 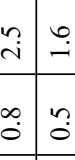 }} & \multirow{2}{*}{\begin{tabular}{|l|}
$\dot{i}$ \\
\\
\\
$\dot{O}$
\end{tabular}} & \multirow{2}{*}{\multicolumn{2}{|c|}{ 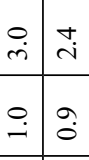 }} & \multirow{2}{*}{\multicolumn{2}{|c|}{\begin{tabular}{l|l}
$\tilde{i}$ & $\stackrel{i}{i}$ \\
$\infty$ & $\hat{0}$ \\
$\dot{0}$ &
\end{tabular}}} & \multirow{2}{*}{\begin{tabular}{|l|}
$m$ \\
$m$ \\
$m$ \\
$m$
\end{tabular}} & \multirow{2}{*}{\multicolumn{2}{|c|}{ 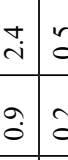 }} \\
\hline & & & & & & & & & & & & & & \\
\hline & & $\bar{\partial}$ & 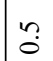 & $\hat{0}$ & $\exists$ & $\stackrel{\circ}{0}$ & $\stackrel{\sim}{=}$ & $\stackrel{ \pm}{ \pm}$ & $\exists$ & $\stackrel{\circ}{-}$ & $\stackrel{0}{-}$ & $\stackrel{0}{-}$ & $\stackrel{\text { Iִ }}{=}$ & \\
\hline & & $\Xi \lesssim$ & 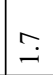 & $\stackrel{\stackrel{i}{i}}{ }$ & $\stackrel{\infty}{i}$ & iี & $\stackrel{m}{i}$ & $\tilde{i}$ & $\stackrel{b}{i}$ & $\vec{i}$ & הָ & $\begin{array}{l}0 \\
i \\
i\end{array}$ & $\vec{i}$ & 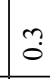 \\
\hline & & $\checkmark$ & $\begin{array}{l}0 \\
\dot{\infty} \\
\dot{\sigma} \\
\end{array}$ & $\begin{array}{l}\vec{f} \\
\text { d. }\end{array}$ & $\begin{array}{l}\vec{\partial} \\
\dot{m}\end{array}$ & in & 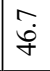 & å. & $\dot{\hat{\sigma}}$ & $\begin{array}{l}0 \\
\dot{y}\end{array}$ & $\stackrel{m}{\xi}$ & $\begin{array}{l}\infty \\
\dot{g} \\
\dot{f}\end{array}$ & $\begin{array}{l}0 \\
\stackrel{1}{n}\end{array}$ & \\
\hline & & $a$ & & $\begin{array}{l}0 \\
8 \\
\end{array}$ & 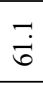 & iि & $\stackrel{m}{n}$ & $\begin{array}{l}\infty \\
\dot{m} \\
\dot{m}\end{array}$ & $\vec{\circ}$ & $\begin{array}{l}0 \\
i n \\
i n\end{array}$ & $\ddot{\circ}$ & $\begin{array}{l}\stackrel{Y}{1} \\
\stackrel{n}{n}\end{array}$ & $\begin{array}{r}\stackrel{+}{\sigma} \\
\stackrel{+}{*}\end{array}$ & 0 \\
\hline$\varepsilon$ & $\triangleright^{\circ}$ & $\tilde{\partial}$ & & $\stackrel{\text { }}{\text { mे }}$ & $\begin{array}{l}\text { ¿ें } \\
\text { mे }\end{array}$ & 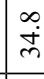 & $\begin{array}{l}n \\
\text { n? } \\
m\end{array}$ & \begin{tabular}{|c}
0 \\
$\dot{m}$ \\
\end{tabular} & $\vec{m}$ & $\begin{array}{l}\dot{q} \\
\dot{m}\end{array}$ & $\begin{array}{l}n \\
n \\
n\end{array}$ & $\mid \begin{array}{l}n \\
0 \\
n\end{array}$ & & $\vec{t}$ \\
\hline & & $\Xi$ & ְे. & है & $\stackrel{+}{\overrightarrow{6}}$ & ชี่ & in & $\mid \begin{array}{l}0 \\
\dot{5}\end{array}$ & : & $\overrightarrow{8}$ & $\mid \begin{array}{l}n \\
0 \\
0 \\
0\end{array}$ & $\mid \begin{array}{l}n \\
0 \\
0\end{array}$ & fi & $\underset{f}{F}$ \\
\hline 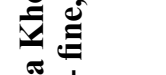 & $x^{0}$ & 3 & & $\stackrel{\stackrel{+}{+}}{\stackrel{\infty}{q}}$ & mे & $\stackrel{\check{q}}{\dot{q}}$ & $\vec{m}$ & $\left|\begin{array}{l}n \\
0 \\
i \\
i\end{array}\right|$ & $\begin{array}{l}n \\
i \\
m\end{array}$ & $\mid \begin{array}{c}\infty \\
\dot{m} \\
\dot{m}\end{array}$ & $\mid \begin{array}{l}\infty \\
\dot{0} \\
\dot{n}\end{array}$ & $\underset{\dot{\sim}}{\stackrel{\sim}{*}}$ & & \\
\hline & & 己 & $\tilde{0}$ & $\stackrel{\circ}{0}$ & $\hat{0}$ & $\hat{0}$ & $\ddot{0}$ & $\because$ & $\stackrel{m}{-}$ & $\exists$ & $\stackrel{0}{0}$ & $\stackrel{m}{-}$ & $\because$ & $\hat{0}$ \\
\hline & & $\approx$ & & $\stackrel{\circ}{\dot{n}}$ & fi & $\stackrel{\circ}{\vec{n}}$ & ?. & $\frac{n}{i}$ & ?ై & $\vec{a}$ & $\begin{array}{l}0 \\
\text { i }\end{array}$ & $\begin{array}{l}m \\
2 \\
i\end{array}$ & $\begin{array}{l}\dot{0} \\
\dot{b}\end{array}$ & O. \\
\hline & $\partial^{\circ}$ & 3 & $\begin{array}{l}\infty \\
0 \\
0\end{array}$ & $\stackrel{\circ}{0}$ & $\stackrel{\circ}{-}$ & $\stackrel{\infty}{0}$ & $\stackrel{\circ}{0}$ & $\stackrel{\circ}{0}$ & $\vec{i}$ & $\stackrel{\infty}{-}$ & $\stackrel{\circ}{-}$ & $\vec{m}$ & $\stackrel{\infty}{\infty}$ & 7 \\
\hline & $\vec{E}$ & $\Xi$ & & $\stackrel{m}{m}$ & $\vec{b}$ & $\begin{array}{l}m \\
\infty \\
m \\
m\end{array}$ & $\overrightarrow{\dot{m}}$ & $\begin{array}{l}\hat{\sigma} \\
\dot{\sigma}\end{array}$ & $\begin{array}{l}\infty \\
\dot{q}\end{array}$ & 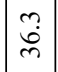 & $\dot{\vec{q}}$ & $\begin{array}{l}0 \\
\dot{q}\end{array}$ & $\stackrel{\dot{p}}{\dot{f}}$ & $\hat{0}$ \\
\hline 5 & & 3 & & రి & $\begin{array}{l}\text { iे } \\
\text { nd }\end{array}$ & $\begin{array}{l}\infty \\
\stackrel{8}{8}\end{array}$ & छे & $\overrightarrow{\dot{n}}$ & $\begin{array}{l}\text { 疋 } \\
\text { Wh }\end{array}$ & $\frac{9}{\dot{0}}$ & $\begin{array}{l}m \\
i n \\
n\end{array}$ & $\begin{array}{l}m \\
\stackrel{n}{n}\end{array}$ & & $\begin{array}{l}\infty \\
\varrho\end{array}$ \\
\hline & & & & ? & $\stackrel{\sim}{n}$ & $\because$ & : & $?$ & $\stackrel{\mathcal{F}}{f}$ & $\stackrel{\mathcal{F}}{\underset{f}{*}}$ & ?3. & $\dot{q}$ & $\tilde{\sigma}$ & 9 \\
\hline & & a & $\stackrel{0}{\circ}$ & $\stackrel{\varrho}{\varrho}$ & $\stackrel{\circ}{i n}$ & $\because$ & $\because$ & $\stackrel{m}{m}$ & $\overrightarrow{6}$ & $\vec{n}$ & $m_{\infty}^{\infty}$ & $\begin{array}{l}\infty \\
i \\
i n\end{array}$ & n & 9 \\
\hline & & $\Xi$ & & $\begin{array}{l}\infty \\
\dot{j} \\
\infty\end{array}$ & $\frac{\partial}{\alpha}$ & $\overrightarrow{1}$ & $\begin{array}{c}\hat{\infty} \\
\infty\end{array}$ & $\begin{array}{l}\not{a} \\
\stackrel{\infty}{0}\end{array}$ & $\vec{\infty}$ & $\hat{\dot{a}}$ & $\begin{array}{c}\dot{a} \\
\dot{\infty}\end{array}$ & 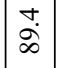 & $\begin{array}{l}\infty \\
\dot{\delta} \\
\infty\end{array}$ & 2 \\
\hline & $\partial^{\circ}$ & $\sqsupset$ & 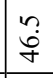 & $\frac{m}{n}$ & $\begin{array}{l}\stackrel{0}{\dot{f}} \\
\dot{f}\end{array}$ & ì & $\begin{array}{l}\infty \\
\dot{\infty} \\
\dot{+} \\
\end{array}$ & $\begin{array}{l}\hat{\dot{q}} \\
\end{array}$ & 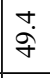 & $\mid \begin{array}{l}\infty \\
\dot{8} \\
\dot{n}\end{array}$ & $\begin{array}{l}0 \\
\dot{\sigma}\end{array}$ & $\begin{array}{l}0 \\
\dot{f} \\
\end{array}$ & & $\stackrel{\mathcal{F}}{\mp}$ \\
\hline & 式 & L & & $\stackrel{+}{\infty}$ & $\stackrel{?}{+}$ & $\stackrel{\circ}{\circ}$ & $\stackrel{2}{\pi}$ & $\begin{array}{l}0 \\
0\end{array}$ & in & $\begin{array}{l}0 \\
\dot{+}\end{array}$ & $\stackrel{m}{\sim}$ & in & छे & $=$ \\
\hline & & $\tilde{\partial}$ & & $\stackrel{+}{\dot{q}}$ & $\vec{\infty}+\overrightarrow{+}$ & लें & 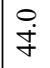 & $\begin{array}{l}m \\
\stackrel{n}{n}\end{array}$ & $\begin{array}{l}\dot{t} \\
\dot{q}\end{array}$ & $\begin{array}{l}0 \\
\dot{J}\end{array}$ & $\stackrel{\ddot{q}}{\dot{q}}$ & $\begin{array}{l}n \\
\dot{q}\end{array}$ & $\begin{array}{l}m \\
\vdots \\
y\end{array}$ & $\stackrel{\sim}{\circ}$ \\
\hline & & 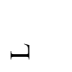 & & $\mid \begin{array}{l}\infty \\
\dot{i}\end{array}$ & $\stackrel{\sim}{\stackrel{N}{\sim}}$ & $\begin{array}{l}\tilde{1} \\
\text { m. }\end{array}$ & त̃ & $\begin{array}{l}-\dot{\infty} \\
\infty\end{array}$ & $\overrightarrow{\mathrm{i}}$ & $\begin{array}{l}\tilde{i} \\
\stackrel{i}{0}\end{array}$ & 苛 & $\begin{array}{l}\infty \\
1 \\
n\end{array}$ & & $\underset{+}{q}$ \\
\hline & 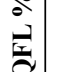 & 工 & & 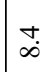 & $\stackrel{m}{+}$ & $\stackrel{\circ}{\circ}$ & $\stackrel{n}{\pi}$ & $\begin{array}{l}0 \\
0\end{array}$ & ñ. & $\begin{array}{l} \\
\stackrel{+}{+}\end{array}$ & $\stackrel{\sim}{\stackrel{r}{r}}$ & $\hat{n}$ & $\hat{\sigma}$ & $=$ \\
\hline & & & & i. & $\stackrel{n}{i}$ & हे & $\stackrel{\circ}{\circ}$ & $\mid \begin{array}{c}m \\
i n \\
i\end{array}$ & $\stackrel{0}{\stackrel{0}{i}}$ & $\vec{a}$ & $\frac{2}{6}$ & $\begin{array}{c}m \\
\infty \\
\infty\end{array}$ & $\stackrel{t}{\stackrel{R}{R}}$ & $\stackrel{\vartheta}{+}$ \\
\hline & & & & $\overrightarrow{\hat{i}}$ & $\stackrel{\text { I }}{\text { I }}$ & స̃. & $\overrightarrow{\mathrm{i}}$ & $\begin{array}{l}0 \\
\dot{\infty} \\
-\end{array}$ & $\begin{array}{l}\infty \\
i \\
i\end{array}$ & $\begin{array}{l}0 \\
\dot{\delta} \\
ن\end{array}$ & 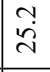 & $\hat{n}$ & & $\stackrel{\infty}{\stackrel{\infty}{+}}$ \\
\hline & & 工 & & $\stackrel{+}{\infty}$ & $\stackrel{m}{q}$ & $\stackrel{\circ}{i}$ & $\stackrel{2}{\simeq}$ & $\ddot{6}$ & in & $\mid \begin{array}{l}\stackrel{0}{+} \\
\end{array}$ & $\stackrel{?}{?}$ & में & aे & $=$ \\
\hline & & $\vec{\sigma}$ & & $\frac{9}{6}$ & & gे & $\grave{i}$ & 芯 & $\stackrel{\stackrel{i}{i}}{i}$ & $\mid \begin{array}{l}* \\
0\end{array}$ & $\mid \begin{array}{l}n \\
\vdots \\
6\end{array}$ & $\begin{array}{l}\dot{a} \\
\stackrel{\infty}{\curvearrowright}\end{array}$ & 点 & $\stackrel{\vec{T}}{+}$ \\
\hline & 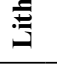 & & & 项 & L & 䟖 & 岸 & $\sum_{\Sigma}^{\infty}$ & $\sum_{\Sigma}^{\infty}$ & $\sum_{\Sigma}^{\infty}$ & $\sum_{\Sigma}^{\infty}$ & $\sum_{\Sigma}^{\infty}$ & & \\
\hline & $\stackrel{\Xi}{ \pm}$ & & & \&̊t & $\stackrel{\mathscr{\gamma}}{\varnothing}$ & $\stackrel{n}{m}$ & $\stackrel{\text { Q }}{\sim}$ & $\stackrel{\infty}{-}$ & $\approx$ & 6 & 8 & $m$ & & \\
\hline & & & & & & & $\overrightarrow{\dot{y}}$ & $\begin{array}{l}\hat{y} \\
\hat{n}\end{array}$ & & $\mid \begin{array}{l}y \\
\vdots \\
2 \\
z\end{array}$ & $\dot{v}$ & $\begin{array}{l}\overrightarrow{\dot{s}} \\
\ddot{z}\end{array}$ & & a \\
\hline
\end{tabular}

\section{DISCUSSION}

\section{Provenance and tectonic setting}

The ternary diagram analyses (Q-F-L, Q-F-R, and Qt-F-L) resulted that the composition of Jagati and Kala Formations' sandstones were plotted almost in the same regions. Garzanti et al. (2007) analyzed the present river sand along the Marsyandi River systems in the western Nepal Himalaya. They revealed the changes in petrographical composition of sands from the Tethys Himalaya to the Lesser Himalayan regions: from the lithic fragment rich sand to the quartz and feldspar rich sand towards downstream (Fig. 3 in Garzanti et al. 2007). The simple comparison with their result suggests the Siwalik sandstones of the Khutia Khola section were contributed mainly from the Lesser Himalaya. As the source of the Lesser Himalaya, these sandstones are rich in quartz grains and poor in rock or lithic fragments which suggest the Kushma (quartzite) and Ranimata (phyllite) Formations, consisting of the Ramgarh Thrust Sheet (DeCelles et al. 2001) are the highly possible source with taking the period of the thrusts activity to the north of our study area (cf. DeCelles et al. 2001; Hyughe et al. 2005). The subrounded to rounded minor chert grains may indicate the water discharge system at that time extended to the Trans-Himalayan region. The obtained compositional data and the sediment source indicated by the sediment grains are, thus, consistent.

Careful comparison of two formations' plots implies that the Jagati Formation sandstones are plotted in the area very slightly toward the Q-R or L axes: the Kala Formation sandstones are slightly richer in feldspar grains. This may be due to the contribution of sand grains from the Higher Himalaya origin. This result matches with the apparent changes in sandstone characteristics: the appearance in "salt and pepper" sandstone in the Kala Formation. The provenance discrimination plots of Dickinson (1985) show that all the Khutia Khola Siwalik sandstones fall in the recycled orogenic field of the Qt-F-L plot, and in the transitional recycled field of the Qm-F-Lt plot (Fig. 6). The plots on the diagram were concentrated along the Qt-L and Qm-Lt lines within the recycled orogenic and transitional recycled areas in the recycled orogen block provenances showing that bulk compositions do not vary significantly within the section. The Qp-Lv-Ls diagram suggests that the Khutia Khola sandstone specimens fall in the field of the collision suture and fold-thrust belt source (Dickinson 1985) (Fig. 7A). The Khutia Khola sandstone composition plots in the Lm-Lv-Ls diagram also suggest the suture zone as their provenance (Ingersoll and Suczek 1979) (Fig. 7C), which is quite consistent with the tectonic setting of the Himalayas. However, the Qm-P-K diagram indicated that the Khutia Khola sandstones' provenance as the continental block with increased maturity (Dickinson 1985) (Fig 7B), which is not consistent with the tectonic setting due to the rich monocrystalline quartz. The abundance in monocrystalline quartz may reflect the presence of granitic and volcanic rocks in the source area (Young 1976): the Dadeldhura granite (Szulc et al. 2006; Bernet et al. 2006) may have contributed on such result in the present case. 


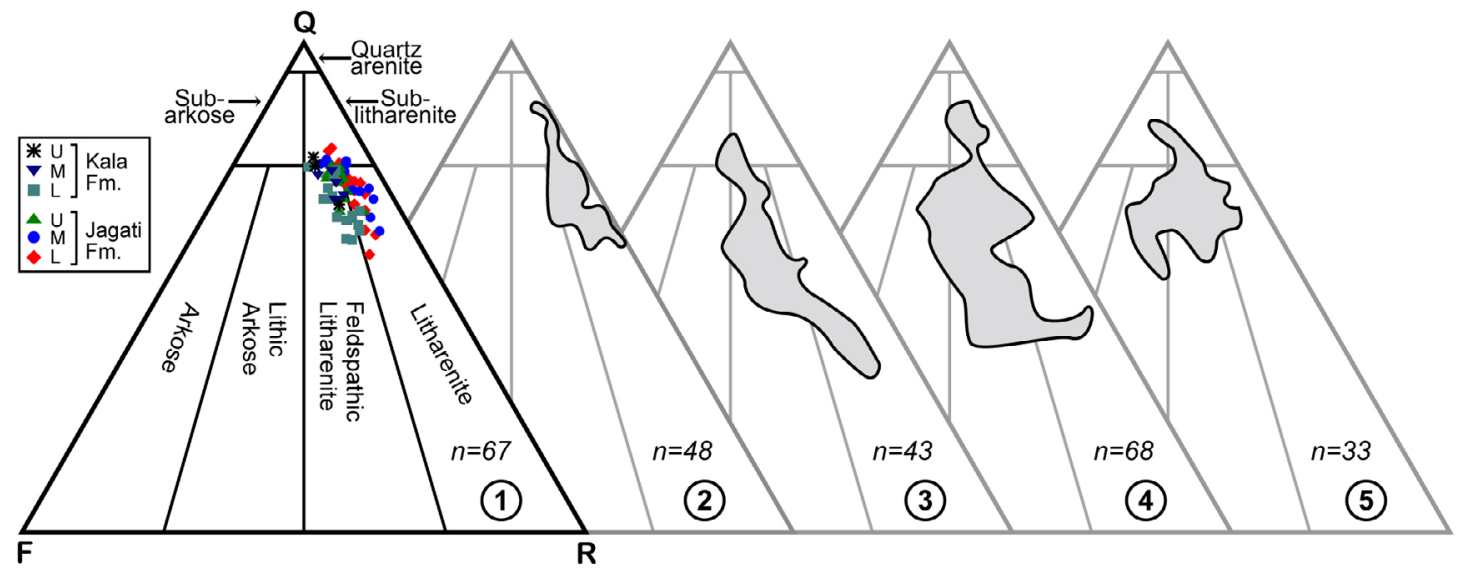

Fig. 5: Comparison of sandstones classification from different sections of the Siwalik Group, Nepal (after Folk 1980). (1) The Khutia Khola section, Far Western Nepal (present study). (2) The Karnali River section, Far Western Nepal (Sigdel and Sakai 2013). (3) The Surai Khola area, Mid Western Nepal (Dhital et al. 1995). (4) The Arung Khola area, West Central Nepal (Tokuoka et al. 1986). (5) The Samari River area, Central Nepal (Tamrakar and Syangbo 2014). Standard plot: Q-F-R (Quartz, feldspar, rock fragments). ' $n$ ' is number of samples.

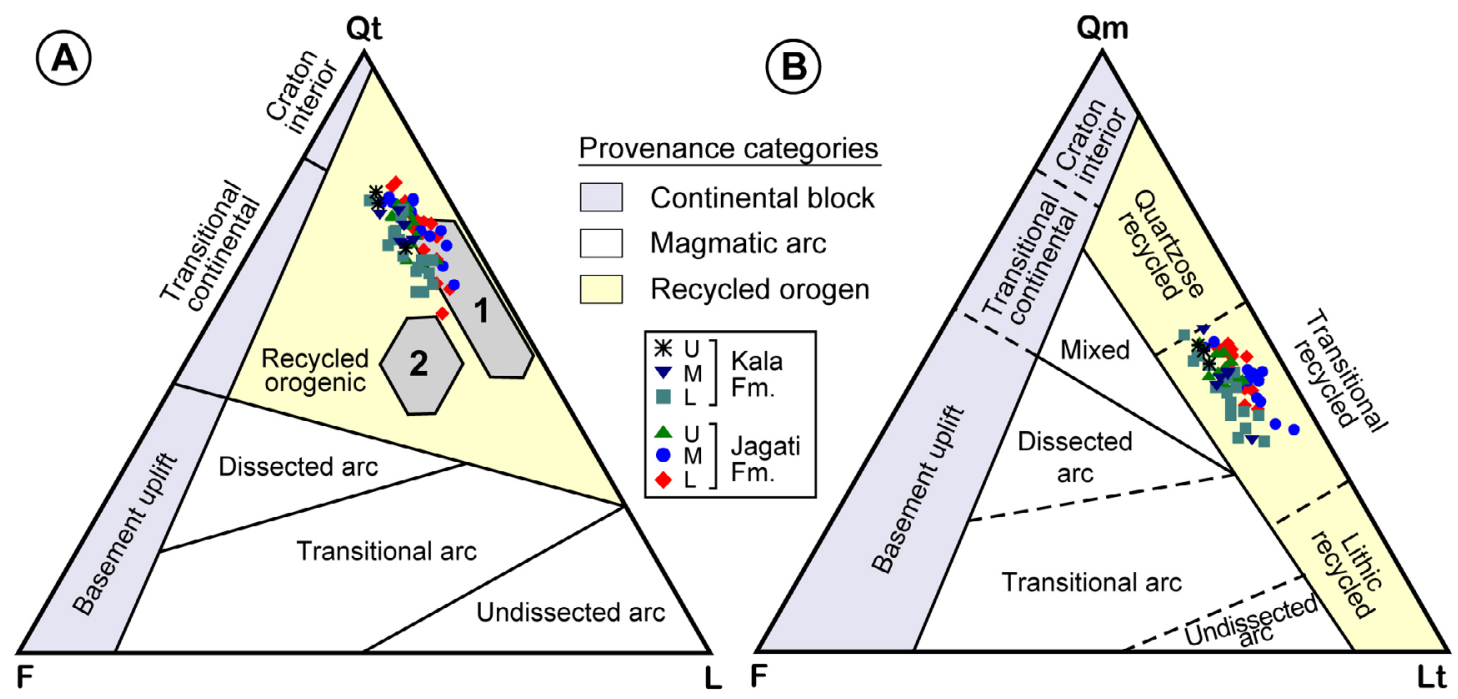

Fig. 6: Ternary diagram for provenance discrimination (after Dickinson 1985). A) Qt-F-L (Quartz, feldspar, lithic fragments). Field 1 = Siwalik Group, Nepal (Surai Khola and Bakiya Khola), and Field 2 = Siwalik Group, Pakistan (Potwar Plateau) (modified from Critelli and Intersoll 1994). B) Qm-F-Lt (Monocrystalline quartz, feldspar, total lithic grains).

\section{Differences in composition of sandstones from other Siwalik sections}

Although the fluvial facies characteristics are different between the Khutia Khola and Karnali River sections, the composition are similar with each other. Slight rich rock fragments in Karnali River section may reflect wider catchment in the Paleo-Karnali River system (Hyughe et al. 2005), which might have been expanded deep in the Himalaya into the Tethys and Trans Himalayan zones.

Comparing the ternary diagrams with other area showed similar composition among Nepal Himalaya, and slightly different one from the Siwaliks of the Potwar Plateau (Pakistan) (Fig. 6A). The detritus derived from volcanic source in their hinterland are the possible cause of the compositional variation in the Potwar Siwalik sediments (Critelli and Ingersoll 1994).
The Khutia Khola sandstones contain less feldspar and lithic fragments relative to Surai Khola and Bakiya Khola sandstones. These compositional variations are possibly due to the different in source lithology in the Lesser Himalaya, such as the Agra Granite and the Palung Granite in west-central, and Amarpur Granite and Kabeli Granite in eastern Nepal.

\section{Climatic indexes}

A biavariant $\log / \log$ plot based on polycrystalline quartz to feldspar plus rock fragments against ratio of total quartz to feldspar allows discrimination on climatic control (Suttner and Dutta 1986). The diagram suggests semi-humid to humid climate during the sedimentation of the Khutia Khola sandstones (Fig. 8A). Tamrakar and Syangbo (2014) have also suggested the semi-humid to humid climate for the Siwalik sandstones in their study area. Plots in the Q-F-R diagram of 

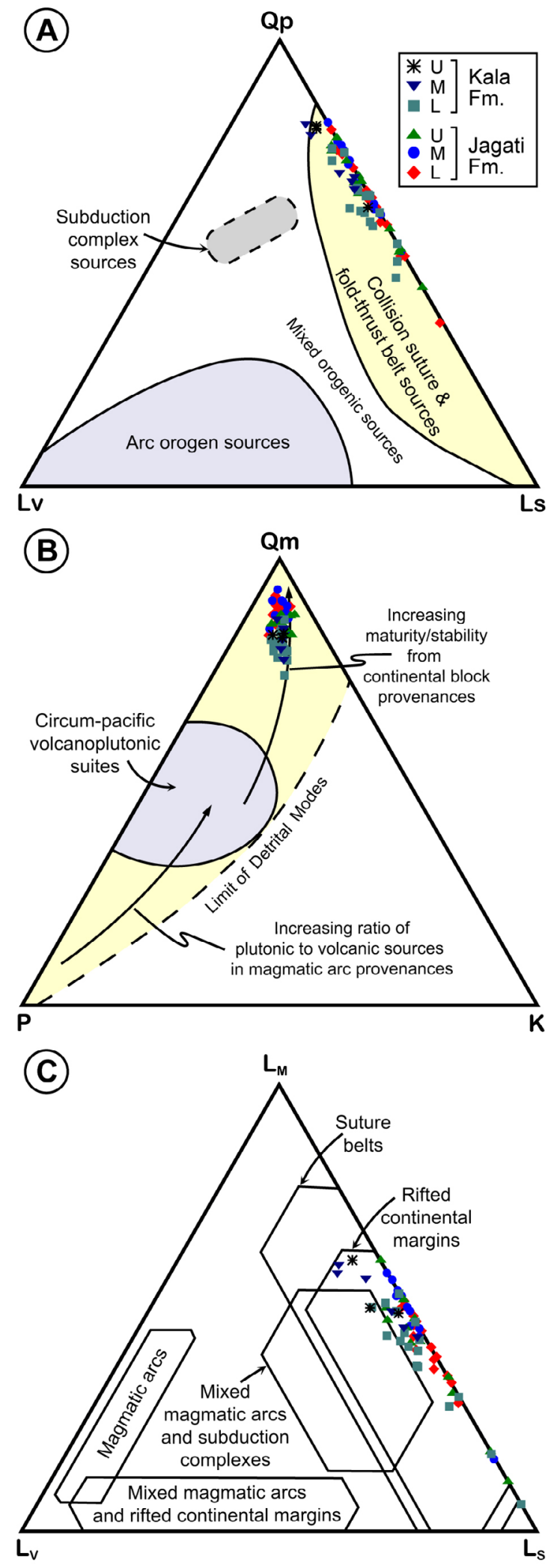

Fig. 7: Ternary diagram for provenance discrimination. (A) Qp-Lv-Ls (Polycrystalline quartz, volcanic lithic fragment, sedimentary lithic fragment) and (B) Qm-P-K (Monocrystalline quartz, plagioclase, K-feldspar) (after Dickinson 1985). (C) Lm-Lv-Ls (metamorphic lithic fragment, volcanic lithic fragment, metamorphic lithic fragment) (after Ingersoll and Suczek 1979).
Suttner et al. (1981) suggest the source of these sandstones is metamorphic rock and sediments were derived under humid climatic condition (Fig. 8B). The Q-F-R diagram by Kundu et al. (2012) have also suggested humid climate for the Siwalik sandstones in Tista valley, India. However, the diagrams (Fig. $8 \mathrm{~A}$ and $8 \mathrm{~B}$ ) are defined for first-cycle sediments and do not discriminate between tectonic settings but can discriminate humid or arid climatic (desert) condition of the source rocks (Suttner et al. 1981; Suttner and Dutta 1986). On these diagrams the Siwalik sediments reflect the semi-humid and humid climatic condition during sedimentation, the result does not contradict with the setting (i.e. metamorphic rock origin; Fig. 8B). In the present case, sediment supply from quartz arenite may have affected slightly on the result: the result appear to be slightly overestimated. The Khutia Khola sandstones contain altered to less-altered, angular to sub-angular feldspars which also indicate burial under humid climatic condition (Basu et al. 1975; Pettijohn et al. 1987). Weltje et al. (1998) proposed a diagram to determine the climatic conditions and the relief in which clastic sediments are deposited. This diagram was constructed by means of current observations in various basins and compares the relationship of the $\log (\mathrm{Q} / \mathrm{F})$ or $\log (\mathrm{Q} / \mathrm{R})$, where $\mathrm{Q}$ stands for the quartz content, $\mathrm{F}$ for the feldspar content and R for the rock-fragment content (Weltje 1994). The use of both logratios in a single diagram allows the distinction of parent rock type, weathering history and paleotopography (Weltje et al. 1998). On this diagram, all the Khutia Khola samples plot in the field of weathering index 1, some are near the boundary between weathering index 0 and 1 (Fig. 8C). Most of the samples from the Jagati Formation fall towards metamorphic and sedimentary parentage and the samples from the Kala Formation fall near the boundary between metamorphic/sedimentary and plutonic parentage. These indices indicate that the sediments were mainly derived from high mountains (Higher Himalaya) and moderate hills (Lesser Himalaya) and the effect of climate was very small (0-1) on the sediment compositions (Fig. 8C). Sigdel and Sakai (2013) have also suggested similar result for the Siwalik sandstones in the Karnali River section.

\section{Stratigraphic variation in petrographic modes}

Stratigraphic variations in petrographic modes were plotted to examine the change in mineral distribution against stratigraphy. Carbonate grains are slightly increased from the upper member of the Jagati Formation to lower part of the lower member of the Kala Formation (Fig. 9A). This increase was possibly due to the Lesser Himalaya (Chirouze et al. 2012). The increment of carbonate grains resembles with the change in river system from FA1 to FA2 which is around 13.5 Ma. Sigdel and Sakai (2013) also found similar trend of carbonate distribution in the Karnali River section. Similarly, $\mathrm{Nd}$ isotopes study also showed more negative values after 13.0 Ma from the Karnali River section (Huyghe et al. 2001, 2005). The feldspar grains remain unchanged in the lower part but the proportion increase from the upper member of the Jagati Formation and continue up to the lower member of the Kala Formation (Fig. 9B). The increase in feldspar grains (mainly $\mathrm{K}$-feldspar) in sandstones indicates erosion of granitic rocks 
Fig. 8: (A) Bivariant $\log / \log$ plot of $Q p /(F+R)$ (polycrystalline quartz to feldspar plus rock fragments) against $Q t /(F+R)$ (total quartz to feldspar plus rock fragments) (after Suttner and Dutta 1986). (B) Ternary Q-F-R (quartz, feldspar, rock fragments) diagram for the effect of source rock on the composition as a function of climate (after Suttner et al. 1981).
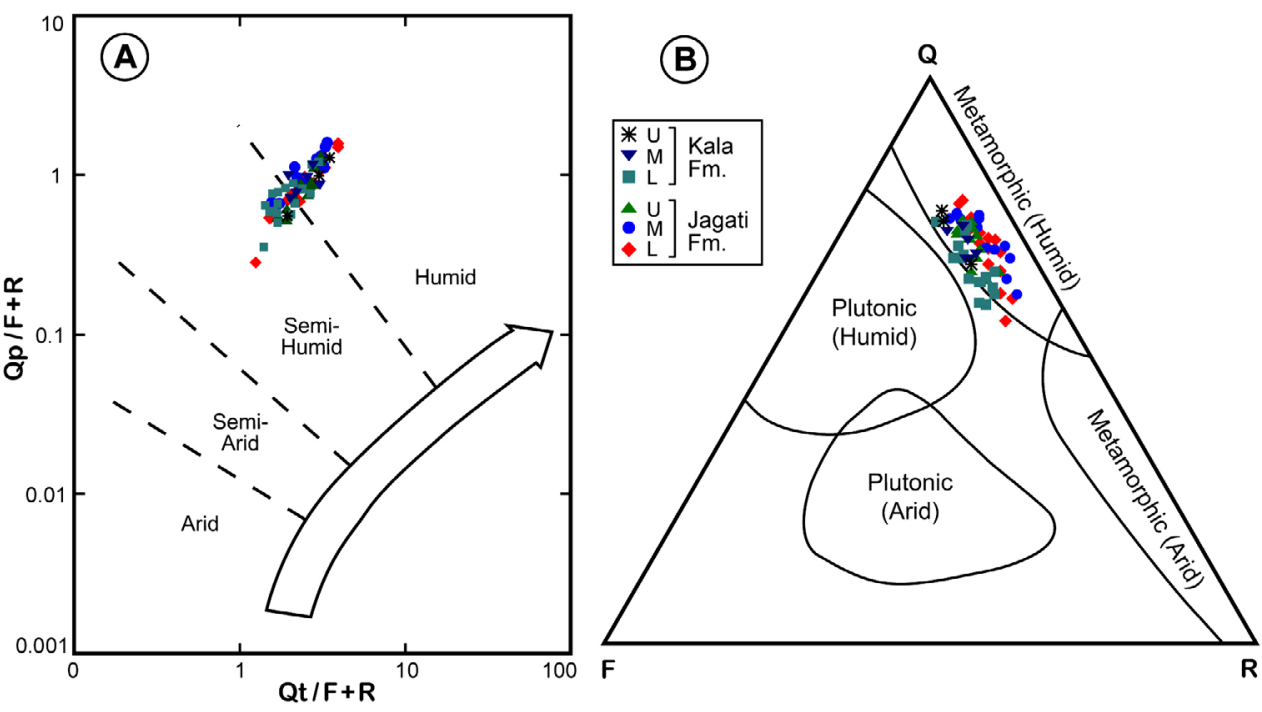

which are mainly derived from Dadeldhura granite (DeCelles et al. 1998). Mica grains do not change significantly but slightly increase upsection in the Kala Formation (Fig. 9C), which indicate that both muscovite and biotite grains are abundant in upsection (cf. DeCelles et al. 1998). The Siwalik Group in the west central Nepal also showed increase in mica content in stratigraphic upsection (Tokuoka et al. 1986; Hisatomi 1990). Similarly, the presence of 'salt and pepper' sandstones in the Kala Formation show increase in feldspar and mica grains after $11.0 \mathrm{Ma}$ and it indicates dominant supply from the Higher Himalayan source in the Middle Siwalik (Sigdel and Sakai 2013). The $\mathrm{Qp} /(\mathrm{F}+\mathrm{R})$ and $\mathrm{Qt} /(\mathrm{F}+\mathrm{R})$ ratios are slightly decreased in stratigraphic upsection with the average values 0.9 and 2.4 for the Jagati Formation, and 0.7 and 2.1 for the Kala Formation, respectively (Table 3 ), which suggest diminishing compositional maturity of sandstones toward stratigraphic upsection (Suttner and Dutta 1986) (Fig. 9D and E).

By combining the petrographic results with other previous studies in the Siwalik Group, we can conclude that the Khutia Khola sediments were mainly derived from the Higher and Lesser Himalaya because no significant changes were observed in the mineralogical composition within the whole section. Presence of low- to high-grade metamorphic lithic fragments even in the the lower part of the Jagati Formation reflect that the Lesser as well as Higher Himalayas provided sediments from the beginning. Similarly, higher content of muscovite and biotite showing 'salt and pepper' appearance sandstones in the Kala Formation (Middle Siwalik) can be related to the unstable tectonic setting and uplift of the Higher Himalaya. The increase in biotite detritus in the Middle Siwalik sandstones indicate dominant supply from the Higher Himalayan source after 11.0 $\mathrm{Ma}$ and this is comparable with the other Siwalik sections in the Nepal but the timing of appearance of the Middle Siwalik is slightly earlier in the Khutia Khola section. This timing is around 9.6 Ma in the Karnali River section in the far western Nepal and around 8.5 to $9.5 \mathrm{Ma}$ in the western and central Nepal. Comparing our petrographical study with Karnali River section (Sigdel and Sakai 2013) together with other studies (Quade et al. 1995; DeCelles et al. 1998; Huyge et al. 2001, 2005; Szulc et al. 2006; Bernet et al. 2006) from all Siwalik

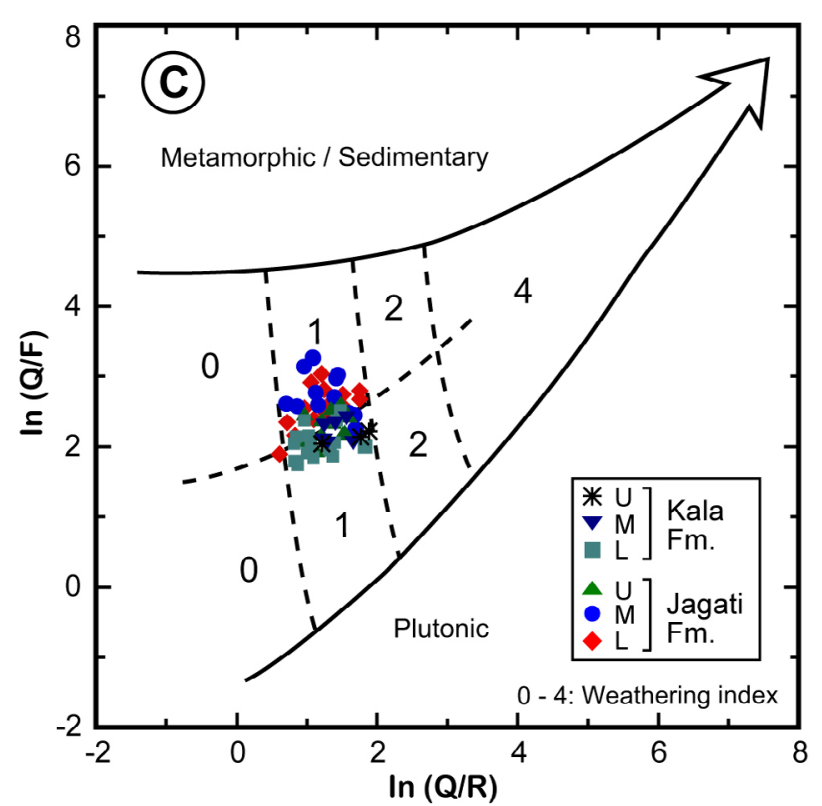

\begin{tabular}{|c|c|c|c|c|}
\hline \multirow{2}{*}{\multicolumn{2}{|c|}{$\begin{array}{l}\text { SEMI-QUANTITATIVE } \\
\text { WEATHERING } \\
\text { INDEX }\end{array}$}} & \multicolumn{3}{|c|}{ PHYSIOGRAPHY (relief) } \\
\hline & & \multirow{2}{*}{$\begin{array}{c}\begin{array}{c}\text { High } \\
\text { (mountains) } \\
0 \\
0\end{array} \\
\end{array}$} & \multirow{2}{*}{$\begin{array}{c}\begin{array}{c}\text { Moderate } \\
\text { (hills) } \\
1\end{array} \\
0\end{array}$} & \multirow{2}{*}{ 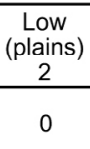 } \\
\hline$\widehat{\widehat{\bar{c}}}$ & $\begin{array}{l}\text { (semi-) arid and } \\
\text { mediterranean }\end{array}$ & & & \\
\hline$\frac{\pi}{6} \cdot \frac{\pi}{0}$ & $\begin{array}{ll}\text { temperate } & \\
\text { subhumid } & 1\end{array}$ & 0 & 1 & 2 \\
\hline 흐 & tropical humid 2 & 0 & 2 & 4 \\
\hline
\end{tabular}

Fig. 8: (cont.) (C) Bivariant log-ratio plot of $Q / F$ (quartz to feldspar) against $Q / R$ (quartz to rock fragments) (after Weltje et al. 1998). Field 1-4 refer to the semi-quantitative weathering indices defined on the basis of relief and climate as indicated in the table.

sections suggest lateral continuity in tectonic uplift of the Himalaya, but an earlier beginning in far western Nepal (cf. Sigdel and Sakai 2013). The earlier appearance of the Middle Siwalik around the Khutia Khola section possibly be related with the activation as well as proximity of the Ramgarh and Dadeldhura Thrusts in the Lesser Himalaya (Foldout 1a. in DeCelles et al. 2001) 

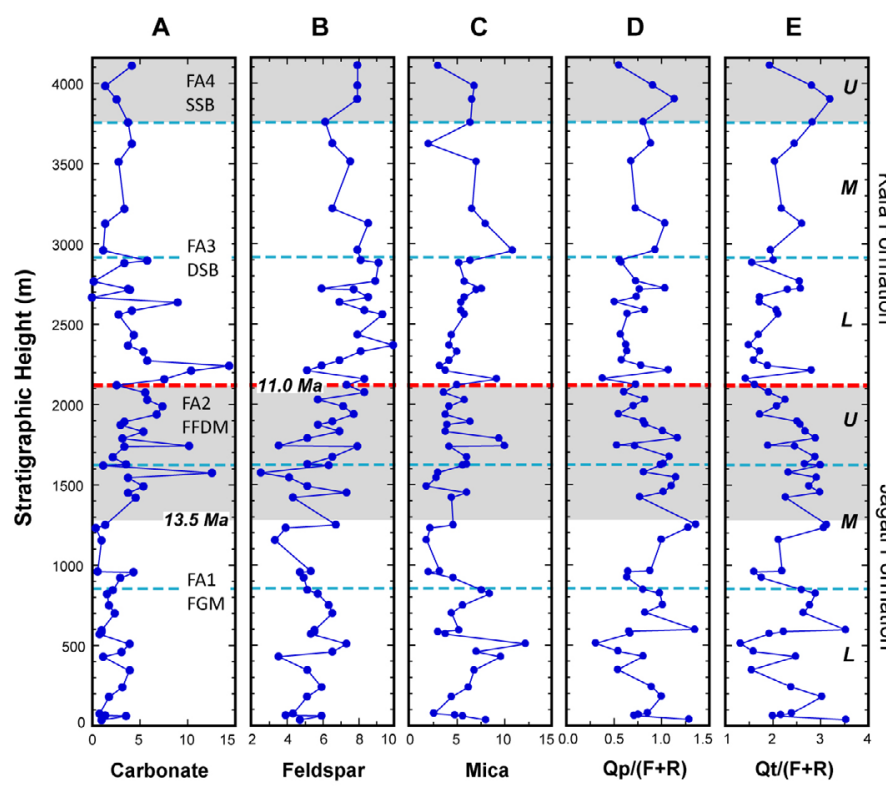

Fig. 9: Plots showing the stratigraphic variations. (A) Carbonate. (B) Feldspar. (C) Mica. (D) $Q p /(F+R)$ (Polycrystalline quartz to feldspar plus rock fragments). (E) $\mathbf{Q t} /(\mathbf{F}+\mathbf{R})$ (Total quartz to feldspar plus rock fragments). Tentative age is denoted with reference of Ojha et al. (2000) (cf. Adhikari and Sakai (2015). Abbreviation: L. lower member, M. - middle member, U. - upper member, FA- facies association, FGM- fine-grained meandering river system, FFDM- flood-flow dominated meandering river system, DSB- Deep sandy braided river system, SSBshallow sandy braided river system.

\section{CONCLUSION}

The major framework grains in the Khutia Khola sandstones are quartz (Q), feldspar (F) and lithic fragments (L; mainly sedimentary and metamorphic), and mica, chlorite, heavy minerals, altered minerals and opaques are present as minor constituent. The average modal compositions of the Jagati and Kala Formations are Q70F7L23 and Q68F10L22, respectively, and overall average composition is Q70F8L22. Petrographically, these sandstones are lithic arenites $(30 \%)$ to sub-litharenites (70\%) and show that the compositions are similar with other Siwalik sandstones.

Provenance analysis indicates that sediments were mainly derived from recycled orogen and fall in transitional recycled block in Qm-F-Lt diagram. The Qp-Lv-Ls and Lm-Lv-Ls diagrams suggest that the samples are from collision suture and fold-thrust belt source. The overall stratigraphic trend of the lithic components shows that metamorphic lithic grains are increasing and sedimentary lithic grains are decreasing in upsection, and the Lesser and Higher Himalayas are the mixed sources of these Siwalik sandstones.

Abivariant plot of $\mathrm{Qp} /(\mathrm{F}+\mathrm{R})$ against $\mathrm{Qt} /(\mathrm{F}+\mathrm{R})$ suggests semihumid to humid climate during sedimentation of the Khutia Khola sandstones and these sandstones are mineralogically immature as well as diminishing compositional maturity towards stratigraphic upsection. Ternary Q-F-R diagram also suggests the deposition under humid climatic condition and metamorphic rock as source. Bivariant $\log / \log$ plot of $(\mathrm{Q} / \mathrm{F})$ and $(\mathrm{Q} / \mathrm{R})$ supports subhumid climate during deposition and these sediments were mainly derived from high mountains (Higher Himalaya) and moderate hills (Lesser Himalaya). The earlier appearance of the Middle Siwalik containing the detrital grains derived from the Higher Himalaya than the other Siwalik sections in Nepal indicates the earlier uplift of that source area and enhanced erosion in the far-western Nepal Himalaya.

\section{ACKNOWLEDGEMENTS}

We thank the Ministry of Education, Culture, Science and Technology of Japan for providing a MEXT scholarship for the study to the first author. We are indebted to B.P. Roser for his guidance during point-counting as well as manuscript writing and to A. P. Gajurel, P. D. Ulak and A. Sigdel for their valuable suggestions for the fieldwork. We also thank to L. Pandey, R. D. Joshi and K. South for their assistance during our fieldwork.

\section{REFERENCES}

Adhikari, S. K., 2014, Geology and variations in fluvial facies and geochemistry of the Neogene Siwalik Group, Khutia Khola area, Nepal Himalaya. Unpublished Master's thesis, Shimane Univ. (Unpublised), 109 p.

Adhikari, S. K., and Sakai, T., 2015, Lithostratigraphy of the Siwalik Group, Khutia Khola section, Far Western Nepal Himalaya. Jour. Nepal Geol. Soc., v. 49, pp. 29-39.

Auden, J. B., 1935, Traverses in the Himalaya. Records of the Geol. Survey India. v. 69, pp. 123-167.

Basu, A., 1976, Petrology of Holocene fluvial sand derived from plutonic source rocks, implication to plaeoclimatic interpretations. Jour. Sed. Petrol., v. 46, pp. 694-709.

Basu, A., Young, S. W., Suttner, L. J., James, W. C., and Mack., G. H., 1975, Re-evaluation of the use of undulatory extinction and polycrystalline in the detrital quartz for provenance interpretation. Jour. Sed. Petrol., v. 45, pp. 873-882.

Bernet, M., Van der Beek, P., Pik, R., Huyghe, P., Mugnier, J. L., Labrin, E., and Szulc, A.G., 2006, Miocene to recent exhumation of central Himalaya determined from combined detrital zircon fission-track and $\mathrm{U} / \mathrm{Pb}$ analysis of Siwalik sediments, western Nepal. Basin Research, v. 18, pp. 393-412.

Blatt, H., Middleton, G. V., and Murray, R. C., 1980, Origin of Sedimentary Rocks. Prentice Hall Inc., 87 p.

Burbank, D. W., Beck, R. A., and Mulder, T., 1996, The Himalayan Foreland: Asian Tectonics. Cambridge Univ. Press, pp. 149188.

Chirouze, F., Bernet, M., Huyghe, P., Erens V., Dupont-Nivet, G., and Senebier, F., 2012, Detrital thermochronology and sediment petrology of the middle Siwaliks along the Muksar Khola section in eastern Nepal. Jour. Asian Earth Sci., v. 44, pp. 94106.

Corvinus, G., and Nanda, A. C., 1994, Stratigraphy and paleontology of the Siwalik group of Surai Khola and Rato Khola in Nepal. N. Jb. Geol. Palaont. Abh., v. 191, pp. 25-68.

Critelli, S., and Ingersoll, R. V., 1994, Sandstone petrography and provenance of the Siwalik Group (northwestern Pakistan and western-southeastern Nepal). Jour. Sed. Res., v. A64(4), pp. 815-823. 
Critelli, S., Le Pera, E., and Ingersoll, R. V., 1997, The effects of source lithology, transport, deposition and sampling scale on the composition of southern California sand. Sediment., v. 44, pp. 653-671.

Crook, K. A. W., 1974, Lithogenesis and geotectonics, the significance of compositional variations in flysch arenites (graywackes). In: Dott, R. H. and Saver, R. H. (Eds.), Modern and Ancient Geosynclinal Sedimentation. Soc. Econ. Paleont. Mineral. Spec. Pub. 91, pp. 304-310.

DeCelles, P. G., Gehrels, G. E., Quade, J., Ojha, T. P., Kapp, P. A., and Upreti, B. N., 1998, Neogene foreland basin deposits, erosional unroofing, and the kinematic history of the Himalayan foldthrust belt, western Nepal. Geol. Soc. Am. Bull., v. 110, pp. 2-21.

DeCelles, P. G., Robinson, M. D., and Quade, J., 2001, Stratigraphy, structure, and tectonic evolution of the Himalayan fold-thrust belt in western Nepal. Tectonics, v. 20, pp. 487-509.

Dhital, M. R., Gajurel, A. P., Pathak, D., Paudel, L. P., and Kizaki, K., 1995, Geology and structure of the Siwaliks and Lesser Himalaya in the Surai Khola-Bardanda area, Mid Western Nepal. Bull. Dept. Geol. Tribhuvan Univ., v. 4, pp. 1-70.

Dickinson, W. R., 1970, Interpreting detrital modes of greywacke and arkose. Jour. Sed. Petrol., v. 40, pp. 695-707.

Dickinson, W. R., 1985, Interpreting provenance relations from detrital modes of sandstones. In: Zuffa, G. G. (Ed.), Provenance of Arenites: North Atlantic Treaty Organization. Advanced Study Institute Series 148, Reidel Publishing Company, pp. 333-361.

Dickinson, W. R., and Suczek, C. A., 1979, Plate tectonics and sandstone compositions. American Assoc. Petrol. Geol. Bull, v. 63 , pp. 2164-2182.

Dickinson, W. R., Beard, L. S., Brakenridge, G. R., Erjavec, J. L., Ferguson, R. C., Inman, K. F., Knepp, R. A., Lindberg, F. A., and Ryberg, P. T., 1983, Provenance of North American Phanerozoic sandstone in relation to tectonic setting. Geol. Soc. Am. Bull., v. 94, pp. 222-235.

Folk, R. L., 1980, Petrology of Sedimentary Rocks. Austin, Texas, Hemphill, $182 \mathrm{p}$.

Gansser, A., 1964, Geology of the Himalayas. Interscience, London, $289 \mathrm{p}$.

Garzanti, E., Vezzoli, G., Ando, S., Lave, J., Attal, M., France-Lanord, C., and DeCelles, P., 2007, Quantifying sand provenance and erosion (Marsyandi River, Nepal Himalaya). Earth Planet. Sci. Lett., v. 258, pp. 500-515.

Gautam, P., and Fujiwara, Y., 2000, Magnetic polarity stratigraphy of Siwalik Group sediments of the Karnali River section in western Nepal. Geophys. Jour. Intern., v. 142, pp. 812-824.

Glennie, K. W., and Ziegler, M. A., 1964, The Siwalik Formations of Nepal. Int.. Geol. Congr., 22, Delhi, v. 25, pp. 82-95.

Hagen, T., 1969, Report on the Geological Survey of Nepal: Preliminary Reconnaissance. Denkschr. Naturw. Ges. v. 86, $185 \mathrm{p}$.

Hisatomi, K., 1990, The sandstone petrology and the provenance of the Churia Group in the Arung Khola - Binai Khola area, west central Nepal. Bulletin Fac. Edu. Wakayama Univ. Nat. Sci., v. 39, pp. 5-29.

Huyghe, P., Galy, A., Mugnier, J. L., and France-Lanord, C., 2001, Propagation of the thrust system and erosion in the Lesser Himalaya: Geochemical and sedimentological evidence. Geology, v. 29, pp. 1007-1010.

Huyghe, P., Mugnier, J. L., Gajurel, A. P, and Decaillau, B., 2005,
Tectonic and climatic control of the changes in the sedimentary record of the Karnali river section (Siwalik of western Nepal). The Island Arc, v. 14, pp. 311-327.

Ingersoll, R. V., and Suczek, C. A., 1979, Petrology and Provenance Neogene sand from Nicobar and Bengal fans. DSDP site 211 and 218. Jour. Sed. Petrol., v. 49, pp. 1217-1228.

Kundu, A., Matin, A., and Mukul, M., 2012, Depositional environment and provenance of Middle Siwalik sediments in Tista Valley, Darjiling District, Eastern Himalaya, India. Jour. Earth System Sci., v. 121(1), pp. 73-89.

Medlicott, H. B., 1875, Note on the geology of Nepal. Rec. Geol. Surv. India., v. 8, pp. 93-101.

Mugnier, J. L., Leturmy, P., Mascle, G., Huygle, P., Chalaron, E., Vidal, G., Husson, L., and Delcaillau, B., 1999, The Siwalik of Western Nepal: I. geometry and kinematics. Jour. Asian Earth Sci., v. 17 , pp. $629-642$.

Nakata, T., 1989, Active faults of the Himalaya of India and Nepal. Geol. Soc. America Special Paper, v. 232, pp. 243-264.

Nakayama, K., and Ulak, P. D., 1999, Evolution of fluvial style in the Siwalik Group in the foothills of the Nepal Himalaya. Sediment. Geol., v. 125, pp. 205-224.

Ojha, T. P., Butler R. F., Quade, J., DeCelles, P. G., Richards, D., and Upreti, B. N., 2000, Magnetic polarity stratigraphy of the Neogene Siwalik Group at Khutia Khola, far western Nepal. Jour. Geol. Soc. Am. Bull., v. 112, pp. 424-434.

Pettijohn, F. J., 1975, Sedimentary Rocks, 3rd ed. Harper \& Tow, New York, $628 \mathrm{p}$.

Pettijohn, F. J., Potter, P. E., and Siever, R., 1987, Sand and sandstone, 2nd ed. Springer-Verlag, $533 \mathrm{p}$.

Pilgram, G. E., 1913, The correlation of the Siwaliks with mammal horizons of Europe. Rec. Geol. Sur. In., v. 43 (4), pp. 264-326.

Prakash, B., Sharma, R. P., and Roy, A. K., 1980, The Siwalik Group (molasses) sediments shed by collision of continental plates. Sediment. Geol., v. 25, pp. 127-159.

Quade, J., Cater, J. M. L, Ojha, T. P., Adam J., and Harrison, T. M., 1995, Late Miocene environmental change in Nepal and the northern Indian subcontinent: Stable isotope evidence form paleosols. Geol. Soc. Am. Bull., v. 107, pp. 1381-1297.

Robinson, D. M., DeCelles, P. G., and Copeland, P., 2006, Tectonic evolution of the Himalaya thrust belt in western Nepal: Implication for channel flow models. Jour. Geol. Soc. Am. Bull., v. 118, pp. 868-885.

Sah, R. B., Ulak, P. D., Gajurel, A. P., and Rimal, L. N., 1994, Lithostratigraphy of the Siwalik sediments of the AmlekhganjHetauda area, sub-Himalaya of Nepal. Him. Geol. v. 15, pp. $37-48$.

Sharma, C. K., 1973, Geology of Nepal. Education Enterprises, Kathmandu. 164 p.

Sharma, S. R., Subedi, D. N., KC, S. B., Khanal, R. P., and Tripathi, G. N., 2007, Geological Map of Petroleum Exploration Block-1, Dhangadi, Far Western Nepal (Scale: 1:250,000). Petroleum Exploration Promotion Project, Department of Mines and Geology, Kathmandu, Nepal.

Sigdel, A., and Sakai, T., 2013, Petrography of Miocene Siwalik Group sandstones, Karnali River section, Nepal Himalaya: Implications for source lithology and tectonic setting. Jour. Nepal Geol. Soc., v. 46, pp. 95-110.

Sigdel, A., and Sakai, T., 2016, Sedimentary facies analysis of the fluvial systems in the Siwalik Group, Karnali River section, Nepal Himalaya, and their significance for understanding the paleoclimate and Himalayan tectonics. Jour. Nepal Geol. Soc., v. 51, pp. 11-26. 
Sigdel, A., Sakai, T., Ulak, P. D., Gajurel, A. P., and Upreti, B. N., 2011, Lithostratigraphy of the Siwalik Group, Karnali River section, far-west Nepal Himalaya. Jour. Nepal Geol. Soc., v. 43, pp. 83-101.

Suttner, L. J., and Dutta, P. K., 1986, Alluvial sandstone composition and paleoclimate. 1. Framework mineralogy. Jour. Sed. Petrol, v. 56, pp. 329-345.

Suttner, L. J., Basu, A., and Mack, G. H., 1981, Climate and the origin of quartz arenite. Jour. Sed. Petrol, v. 51, pp. 1235-1244.

Szulc, A. G., Najman, Y., Sinclair, H., Pringle, M., Bickle, M., Chapman, H., Garzanti, E., Ando, S., Huyghe, P., Mugnier, J. L., Ojha, T. P., and DeCelles, P. G., 2006, Tectonic evolution of the Himalaya constrained by detrital 40Ar-39Ar, Sm-Nd and petrographic data from the Siwalik foreland basin succession, SW Nepal. Basin Research, v. 18, pp. 375-391.

Tamrakar, N. K., Yokota, S., and Shrestha, S. D., 2003, Petrography of the Siwalik sandstones, Amlekhganj-Suparitar area, central Nepal Himalaya. Jour. Nepal Geol. Soc., v. 28, pp. 41-56.

Tamrakar, N. K., and Syangbo, D. K., 2014, Petrography and provenance of the Siwalik Group sandstones from the Main Boundary Thurst region, Samari River area, central Nepal, Sub-Himalaya. Boletin de Geologia, v. 36(2).

Tokuoka, T., Takayasu, K., Yoshida, M., and Hisatomi, K., 1986, The Churia (Siwalik) Group of the Arung Khola area, West Central Nepal. Mem. Fac. Sci. Shimane Univ., v. 20, pp. 135-210.

Tokuoka, T., Takeda, S., Yoshida, M., and Upreti, B. N., 1988, The Churia (Siwalik) Group in the western part of the Arung Khola area, west central Nepal. Mem. Fac. Sci. Shimane Univ., v. 22, pp. 131-140.

Ulak, P. D., 2004, Evolution of fluvial system in Siwalik Group of Chatara-Barahakshetra area, east Nepal Himalaya. Jour. Nepal Geol. Soc., v. 30, pp. 67-74.

Ulak, P. D., 2009, Lithostratigraphy and late Cenozoic fluvial styles of Siwalik Group along Kankai River section, East Nepal Himalaya. Bull. Dep. Geol., Tribhuvan Univ., v. 12, pp. 63-74.

Ulak, P. D., and Nakayama, K., 1998, Lithostratigraphy and evolution of the fluvial style in the Siwalik Group in the Hetauda-Bakiya
Khola area, Central Nepal. Bull. Dep. Geol., Tribhuvan Univ., v. 6, pp. 1-14.

Ulak, P. D., and Nakayama, K., 2001, Neogene fluvial systems in the Siwalik Group along the Tinau Khola section, west central Nepal Himalaya. Jour. Nepal Geol. Soc., v. 25, (Sp. Issue), pp. 111-122.

Ullah, K., Arif, M., and Shah, T. M., 2006, Petrography of sandstones from the Kamlial and Chinji Formations, southwestern Kohat Plateau, NW Pakistan: Implications for source lithology and paleoclimate. Jour. Himalayan Earth Sci., v. 39, pp. 1-13.

Upreti, B. N. 1999, An overview of the stratigraphy and tectonics of the Nepal Himalaya. Jour. Asian Earth Sci., v. 17, pp. 577-606.

Upreti, B. N., and Le Fort, P., 1999, Lesser Himalayan crystalline nappes of Nepal: Problems of their origin, in Macfarlane, A., Sorkhabi, R. B., and Quade, J., (Eds.), Himalaya and Tibet: Mountain Roots to Mountain Tops. Geol. Soc. Am., v. 328 (Sp. Paper), pp. 225-238.

Wadia, D. N., 1957, Geology of India. Macmillan Pub., London, 536 p.

Weltje, G. J., 1994, Provenance and dispersal of sand-sized sediments: reconstruction of dispersal patterns and sources of sand-size sediments by means of inverse modeling techniques. $\mathrm{PhD}$ thesis, Geologica Ultraiectina.

Weltje, G. J., Meijer, X. D., and De Boer, P. L., 1998, Stratigraphic inversion of siliciclastic basin fills: a note on the distinction between supply signals resulting from tectonic and climatic forcing. In: Hovius, N., Leeder, M. (Eds.), Thematic Set on Sediment Supply to Basins. Basin Research, v. 10, pp. 129-153.

Weltje, G. J., 2002, Quantitative analysis of detrital modes: statistically rigorous confidence region in ternary diagrams and their use in sedimentary petrology. Earth Sci. Rev., v. 57, pp. 211-253.

Yoshida, M., and Arita, K., 1982, On the Siwaliks observed along some routes in Central Nepal. Jour. Nepal Geol. Soc., v. 2, (Sp. Issue), pp. 51-58.

Young, S. W., 1976, Petrographic textures of detrital polycrystalline quartz as an aid to interpreting crystalline source rocks. Jour. Sed. Petrol., v. 46, pp. 595-603. 